\title{
The Hubble Deep Field South Flanking Fields
}

\author{
Ray A. Lucas, ${ }^{1}$ Stefi A. Baum, ${ }^{1}$ Thomas M. Brown, ${ }^{1}$ Stefano Casertano, ${ }^{1,6}$ Chris \\ Conselice, ${ }^{1}$ Duilia de Mello, ${ }^{1}$ Mark E. Dickinson, ${ }^{1}$ Henry C. Ferguson, ${ }^{1}$ Andrew S. \\ Fruchter, ${ }^{1}$ Jonathan P. Gardner, ${ }^{3}$ Diane Gilmore, ${ }^{1}$ Rosa A. González-Lópezlira, ${ }^{1}$ Inge \\ Heyer, ${ }^{1}$ Richard N. Hook, ${ }^{2}$ Mary Elizabeth Kaiser, ${ }^{3,4}$ Jennifer Mack, ${ }^{1}$ Russell Makidon, ${ }^{1}$ \\ Crystal L. Martin, ${ }^{5}$ Max Mutchler, ${ }^{1}$ T. Ed Smith, ${ }^{1}$ Massimo Stiavelli, ${ }^{1,6}$ Harry I. Teplitz,${ }^{3}$ \\ Michael S. Wiggs, ${ }^{1}$ Robert E. Williams, ${ }^{1}$ and David R. Zurek ${ }^{1}$
}

\begin{abstract}
As part of the Hubble Deep Field South program, a set of shorter 2-orbit observations were obtained of the area adjacent to the deep fields. The WFPC2 flanking fields cover a contiguous solid angle of 48 square arcminutes. Parallel observations with the STIS and NICMOS instruments produce a patchwork of additional fields with optical and near-infrared $(1.6 \mu \mathrm{m})$ response. Deeper parallel exposures with WFPC2 and NICMOS were obtained when STIS observed the NICMOS deep field. These deeper fields are offset from the rest, and an extended low surface brightness object is visible in the deeper WFPC2 flanking field. In this data paper, which serves as an archival record of the project, we discuss the observations and data reduction, and present SExtractor source catalogs and number counts derived from the data. Number counts are broadly consistent with previous surveys from both ground and space. Among other things, these flanking field observations are useful for defining slit masks for spectroscopic follow-up over a wider area around the deep fields, for studying large-scale structure that extends beyond the deep fields, for future supernova searches, and for number counts and morphological studies, but their ultimate utility will be defined by the astronomical community.
\end{abstract}

\footnotetext{
${ }^{1}$ Space Telescope Science Institute, 3700 San Martin Drive, Baltimore, MD 21218

${ }^{2}$ Space Telescope - European Coordinating Facility, European Southern Observatory, Karl-SchwarzschildStrasse 2, D-85748, Garching bei München, Germany

${ }^{3}$ Laboratory for Astronomy and Solar Physics, Code 681, Goddard Space Flight Center, Greenbelt, MD 20771

${ }^{4}$ Department of Physics \& Astronomy, Johns Hopkins University, Baltimore, MD 21218

${ }^{5}$ University of California at Santa Barbara, Department of Physics, Santa Barbara, CA 93106

${ }^{6}$ On assignment from the Space Sciences Division of the European Space Agency
} 
Subject headings: catalogs — cosmology: observations — galaxies: evolution — galaxies: statistics — galaxies: photometry — surveys.

\section{Introduction}

The history of the Hubble Deep Field-North (HDF-N, Williams et al. 1996) represents an example of the benefits obtained from technological advances and observing from space. Following the first HST servicing mission, relatively deep observations of the cluster CL0939+4713 at $\mathrm{z}=0.4$ (Dressler et al. 1994) and the cluster around 3C324 at $\mathrm{z}=1.2$ (Dickinson et al. 1995), as well as observations of field galaxies from the HST Medium Deep Survey (Griffiths et al. 1996) demonstrated that the Hubble Space Telescope with its restored optical capability could yield images of distant galaxies with unprecedented clarity, revealing many faint galaxies and a wealth of morphological detail in objects observed. The Hubble Deep Field-North was the culmination of these early efforts. In order to provide a similar opportunity for follow-up by observatories in the southern hemisphere, and to provide comparison along a different line of sight another deep-field campaign was carried out in October, 1998. For this second field, a line-of-sight to a QSO at redshift $z=2.2$ was chosen, offering the opportunity to correlate properties of the intergalactic medium to the density of surrounding galaxies. New instruments on HST also provided the opportunity for ultraviolet and infrared imaging on the second campaign, as well as providing spectroscopy and deep unfiltered optical imaging. Scientific results from the Hubble Deep Fields are reviewed by Ferguson, Dickinson, \& Williams (2000). Further details of the HDF-S observations can be found in Williams et al. (2000), Casertano et al. (2000), Gardner et al. (2000), and Fruchter et al (2002).

The Hubble Deep Field-North (HDF-N, Williams et al. 1996) program included a set of shallower Wide Field Planetary Camera 2 (WFPC2) observations immediately adjacent to the deep WFPC2 field. These fields were included for the benefit of observers who could use the objects in those fields as additional targets when obtaining spectroscopic observations of the primary objects in the Deep Field itself (e.g. Cohen et al. 2000). The HDF-N flanking fields (FFs) have proven to be scientifically useful in a variety of ways. They have provided morphological and photometric data for targets of ground-based spectroscopy. They have contributed to studies of Galactic structure via star counts, as well as studies of extragalactic structures on a scale larger than the Deep Field, and have provided detections and morphologies of objects identified in X-ray, radio, infrared, and sub-mm surveys.

Similar flanking field observations were included in the Cycle 7 Hubble Deep Field-South (HDF-S, Williams et al. 2000) campaign which included the newly-available Space Telescope 
Imaging Spectrograph (STIS) and the Near-Infrared Camera Multi-Object Spectrometer (NICMOS) as well as WFPC2. The quasar J2233-606 was centered in the STIS field and observed both spectroscopically and in imaging mode at a variety of wavelengths. NICMOS and WFPC2 provided simultaneous multi-color imaging in nearby parallel deep fields. The availability of STIS and NICMOS in addition to WFPC2 has augmented the scientific utility of the HDF-S FFs, allowing us not only to probe the extended environment of the QSO in the area between the deep fields, but also other "core samples" at somewhat greater distances.

The HDF-S flanking-fields campaign consisted of 27 orbits of observations. Nine contiguous WFPC2 fields were observed for two orbits each, and exposures were taken in parallel with NICMOS and STIS. The remaining nine orbits were invested in a STIS clear-filter image of the NICMOS deep field (STIS-on-NICMOS), accompanied by parallel observations with the other two instruments. This campaign resulted in shallow WFPC2 coverage of a contiguous area stretching between the STIS and NICMOS deep fields, surrounded by a set of offset fields with coverage by a single instrument. The resulting patchwork of observations is illustrated in Figure 1 of Williams et al. (2000).

A version of the STIS-on-NICMOS image which was convolved with the NICMOS PSF, and the catalog derived from that image, will be presented in the paper by Fruchter et al. (2002) on the NICMOS deep field. The unconvolved STIS-on-NICMOS image and the catalog derived from it, which has a somewhat fainter magnitude limit than that derived from the NICMOS PSF-convolved image, will be presented in this paper.

Williams et al. (2000) gives a general overview of the HST observations of the HDF-S, and describe the rationale for adopting the field around QSO J2233-606 for the campaign. The present paper describes the HDF-S flanking field observations and data processing, and presents source catalogs and number counts. In Section 2, we describe the observations, while Sections 3, 4, and 5 describe data reduction and image combination for WFPC2, STIS, and NICMOS FFs, respectively. Astrometry updates and large mosaiced images are covered in Section 6, and in Sections 7 and 8 we present the catalogs and number counts.

\section{Observations}

The HDF-South FF observations constitute the HST program ID 8071, and the observation sequence and detailed observing plan are available at the Space Telescope Science Institute program information web page.

The WFPC2 FFs form a triangle connecting the three deep fields. All observations of the flanking fields were taken at the same orientation. Small shifts of pointing position 
(dithering) were introduced between exposures to aid calibration and to allow improved sampling of the point-spread function. The STIS and NICMOS parallel FFs have little or no overlap with other fields. They are located at greater radial distances from the quasar than most of the WFPC2 FFs, and they reach fainter magnitude limits than the WFPC2 fields. The STIS images are higher resolution than the WFPC2 observations, whereas the NICMOS images are lower. Figure 1 of Williams et al. (2000) shows the locations of the fields and Figure 5 of the same paper displays a mosaic of the HST data of all the HDF-S primary and flanking fields combined.

\subsection{WFPC2 observations}

Nine two-orbit WFPC2 F814W FFs (FF1-FF9) cover roughly 10.5' (East-West) $\times 9.5^{\prime}$ (North-South) diagonally between the STIS, NICMOS, and WFPC2 deep fields, overlapping both STIS and NICMOS deep fields. The exposures were not CR-SPLIT, but were singlydithered exposures (i.e. a single exposure at each dither position) of 1200 or 1300 seconds. The 9-orbit WFPC2 parallels to the STIS-on-NICMOS observations were CR-SPLIT so that there were two images at each dither position, with exposure times of 1100 or 1200 seconds per exposure.

The FF1-FF9 dithering pattern consisted of four images, with one image per dither position, in a bent "Y" spanning 2.5 " in $\mathrm{x}$ and 4.0 " in y. The dithering pattern for the WFPC2 images taken in parallel with the primary STIS-on-NICMOS observations was determined by the dithering pattern for the primary STIS observations of the NICMOS deep field. It consisted of a four-point diagonal line spanning $16^{\prime \prime}$ in both $\mathrm{x}$ and $\mathrm{y}$ for $\mathrm{F} 606 \mathrm{~W}$, and a five-point bent diagonal line spanning $7^{\prime \prime}$ in $\mathrm{x}$ and $6^{\prime \prime}$ in $\mathrm{y}$ for $\mathrm{F} 814 \mathrm{~W}$.

\subsection{STIS observations}

For each of FF1-FF9, STIS observations were made in parallel with the primary instrument, WFPC2. In general, there is only a small overlap between the STIS flanking field observations and other HDF-S data. After guide star acquisition, a CR-SPLIT 1200s exposure was followed by a $1300 \mathrm{~s}$ CR-SPLIT exposure at a new dither (shifted) position. A second orbit of observations immediately followed with two more dithered CR-SPLIT exposures of $1300 \mathrm{~s}$ each for a total of 8 readouts at 4 dither positions. The dither pattern for these nine fields was dictated by the primary WFPC2 observations.

There were an additional 9 orbits of data in 50CCD unfiltered mode taken as primary 
observations with the STIS centered on the NICMOS deep field. These STIS-on-NICMOS observations were made using 9 single orbit-long non-CR-SPLIT exposures at 9 different dither positions, one per orbit, with all but 2 falling in a diagonal line spanning $16^{\prime \prime}$ in both $\mathrm{x}$ and $\mathrm{y}$, with each of the inner 5 points in the line separated from its nearest neighbor in the inner group by $2^{\prime \prime}$ in $\mathrm{x}$ and $\mathrm{y}$, and the outer two points at either end of the diagonal separated from the outermost of the inner group by $4^{\prime \prime}$ in $\mathrm{x}$ and $\mathrm{y}$. Two additional points were offset from the center by $3^{\prime \prime}$ in $\mathrm{x}$ and $2^{\prime \prime}$ in $\mathrm{y}$, and $-3^{\prime \prime}$ in $\mathrm{x}$ and $-2^{\prime \prime}$ in $\mathrm{y}$, respectively.

Observations of two 10th magnitude stars were made before and after the HDF-S campaign to measure the point spread function (PSF) in STIS 50CCD imaging. An accurate measurement of the PSF was necessary for subtraction of the quasar image in the STIS $50 \mathrm{CCD}$ deep field in order to search for the host galaxy or galaxies very close to the target quasar. These PSF images were all short exposures of 20 seconds or less. About half of those planned for 20 September, 1998 were affected by a failure of the guide star acquisition which caused the aperture to remain shut. All the affected exposures were of less than 4 seconds duration, however there were also a number of successful exposures of duration between 0.2 second and 1 second. See Section 2.6 of Gardner et al. (2000) for more details on these PSF observations.

\subsection{NICMOS observations}

Nine two-orbit NICMOS F160W FFs were also taken in parallel with the WFPC2 primary FF observations. These images have little or no overlap with other fields, and their dithering pattern was determined by that of the primary WFPC2 FF observations described above. The nine-orbit F110W and F160W parallels to the STIS-on-NICMOS deep field observations were dithered according to the pattern used for those primary STIS observations. Both F110W and F160W images were taken at each dither position, with the pattern consisting of a nine-point bent diagonal line of $16^{\prime \prime}$ in both $\mathrm{x}$ and $\mathrm{y}$. NIC1 and NIC2 parallel observations were also included with the NIC3 observations for all of the flanking fields, but the internal NICMOS focus was always optimized for the NIC3 camera. The out-of-focus NIC1 and NIC2 images are not scientifically useful and will not be discussed further. 


\section{WFPC2 Data Reduction and Image Combination}

The WFPC2 flanking fields data were reduced in a manner similar to that used for the WFPC2 deep field (see Casertano et al. 2000), but with some differences in detail. Although giving a general description of the process used, we will concentrate most on the differences here, and the reader is referred to Casertano et al. (2000) for more details of the HDF-S WFPC2 data processing. Unless otherwise noted, this paper describes processing done to create the currently available version 2 WFPC2 images. Some improvements have been made since the version 1 images which were released immediately after the HDF-S campaign in 1998. These are briefly described in Section 3.4.

\subsection{Data Reduction}

The initial stages of processing involved inspection of the data and application of the WFPC2 HDF-S calibration pipeline (flat-fielding, dark corrections, etc.). Prior to image combination and cosmic-ray rejection, the sky background in each image was determined from the mode of the pixel values and subtracted from the image.

\subsection{Image Combination}

The HST cameras introduce geometrical distortion and can significantly undersample the point-spread function of the telescope. In order to facilitate the detection of image defects and overcome some of the effects of spatial undersampling, the images described here were dithered, following standard HST procedures, using small offsets between exposures. The resultant images present significant image combination challenges. We have adopted the DRIZZLE method (Fruchter \& Hook 2002) which was originally developed for the HDF-N and successfully used for much HST imaging since, as the basic image combination tool. This allows for geometric distortion correction, optimal pixel weighting, and minimized resolution loss during image coaddition. DRIZZLE and associated tools are available in robust implementations within the STSDAS DITHER package.

The images were thus combined using tasks and algorithms in the STSDAS DITHER package (Fruchter et al. 1997, Koekemoer 2002). Briefly, after sky subtraction, images were filtered to mimimize the impact of cosmic rays, then cross-correlated to determine relative shifts. The Planetary Camera (PC) chip was excluded from the calculation since it has less signal per pixel but the same amount of noise from cosmic rays as the three Wide-Field Camera (WF) chips. 
Once shifts were determined, masks for each image were created to trim off the edges and flag the known bad pixels. Properly mosaiced WFPC2 frames were drizzled with the correct orientation (North up and East to the left) at a scale of $0.8^{\prime \prime}$ per pixel. The edges of the static masks were trimmed, and the first drizzle was performed with a drop-size or "PIXFRAC" of 1.0. (See Fruchter \& Hook 1997 and Fruchter \& Hook 2002 for discussion and a definition of PIXFRAC.) These mosaiced images (4 per FF 1-9 fields) were then combined with IMCOMBINE using MINMAX rejection to produce a median image. This median image was then transformed back to the scale and coordinate system of the original images using the BLOT task.

A cosmic ray mask was then created for each flanking-field image by comparison with the median image. The cosmic-ray rejection algorithm (DRIZ_CR) makes two passes over the image, first rejecting pixels that do not match the median image beyond a fixed threshold ( $4 \sigma$ in our case), then rejecting neighbors that exceed some lower threshold $(3 \sigma)$. Satellites or other fast moving objects which cross the field of view during an observation appear as a linear stripe across the images. To fix this problem, these were identified by eye on the individual exposures, and the regions containing the moving object were added to the mask. These regions were typically 10-20 pixels wide, and extended across the mosaic. Moving objects were blocked from a single exposure in each of FF2, FF3, FF5 and FF9. The second and final drizzle was done on the original images, with a scale of $0.05^{\prime \prime}$ per pixel and a PIXFRAC of 0.7, using the masks of the edges, bad pixels, and cosmic rays. Pixels where the weights equaled zero were set to zero. The final images were converted to counts per second, and world coordinate system parameters in the data headers were updated based on astrometry from the USNO (Zacharias et al. 1998) catalog. The updated coordinates of objects and positions in the images can thus be retrieved via the IRAF task XY2RD or similar tasks.

The F606W and F814W STIS-on-NICMOS WFPC2 images were co-aligned by cross correlating drizzled, cosmic-ray rejected mosaics of each band. The images were then redrizzled using the shifts determined from this cross-correlation. Finally, a large mosaic of FFs 1-9 was also drizzled, and a version including all the deep fields and flanking fields in all the instruments was later drizzled as well. These are discussed further in the context of WFPC2 astrometry and large mosaics in Section 6.1.

The details of Right Ascension (RA) and Declination (Dec) of the centers of the final drizzled images, plus observation dates, filters, number of exposures, and exposure times per WFPC2 flanking field are given in Table 1. Figures 1-5 show WFPC2 Flanking Fields 1-9 and the combined deeper WFPC2 F606W and F814W parallels to the STIS-on-NICMOS observations. Especially of note is an extended low surface brightness object in the deeper 
combined F606W and F814W WFPC2 image shown in the lower half of Figure 5.

Our processing produced both science images and weight images. These are presented in a single mosaic for each field, with all four WFPC2 detectors combined onto the same image plane. The science images were weighted by their exposure time during combination. The PC images had an additional weighting factor: the square of (WF camera DRIZZLE scale/ PC DRIZZLE scale). The final output pixels, both WF and PC, are 0.0498". The final images are expressed in counts per second at a gain of 7 , and have been rotated to have North up (to within about 0.5 degrees from vertical). Since a number of dither positions were combined together for each field, there is variable coverage across the field of view. The image depth decreases near the edges of each field, and in the seam between detectors.

For the version-2 data release, the weight images were scaled so that they represent the inverse of the variance of the observations. The scaling was set so that the variance as predicted in $4 \times 4$ pixel regions of the final weight image matches the variance as actually measured from the same size regions of the background in the final combined object image. Refer to Sections 3.5 and 4.1 of Casertano et al. (2000) and to Fruchter \& Hook (2002) for a detailed description of noise estimates from drizzled weight images.

\subsection{Photometric zeropoints}

The WFPC2 detectors differ slightly in their sensitivity, and we have scaled the input images to the response of the WF3 detector. We adopted the zero points determined for WF3 at gain 7. For the F606W and F814W filters, values of 23.04 and 22.09 were used, respectively. These correspond to the AB magnitude of a source that would produce 1 count per second:

$$
m=-2.5 \times \log _{10}(\mathrm{DN} / \mathrm{EXPTIME})+\mathrm{ZEROPOINT}
$$

The AB magnitude system (Oke 1971) is defined such that a flat-spectrum source (constant $f_{\nu}$ ) would produce the same magnitude in all bands. An alternative system is defined such that Vega has magnitude 0 in all bands. The conversion is VEGAMAG $=$ ABMAG -0.11 for the F606W band and ABMAG - 0.44 for the F814W band. The WFPC2 calibration via photometric standard stars is discussed in the HST WFPC2 data handbook (Baggett et al. 2002). The counts-to-magnitude conversion refers to an "infinite" aperture, defined as having 1.096 times the flux in an aperture with $0.5^{\prime \prime}$ radius. This definition is equivalent to setting the aperture correction between a $0.5^{\prime \prime}$ radius aperture and an infinite aperture to exactly $0.10 \mathrm{mag}$.

For the 2-orbit WFPC2 fields, the estimated limiting magnitude is 26.0 ( $5 \sigma$ in an aper- 
ture of area 0.2 square arcsecond.) For the STIS-on-NICMOS F606W parallel, we similarly thus estimate a limiting magnitude of 27.8, and for the F814W STIS-on-NICMOS parallel, we estimate a limiting magnitude of 27.2 .

\subsection{Current Status and Future Plans}

The version 1 images released shortly after the HDF-S campaign have been replaced by improved version 2 images. These images have improved astrometry (described in greater detail below, in Section 6; the original version 1 astrometry was only good to the accuracy of the Guide Star Catalog, or about 0.5" to 1.0"), coalignment of F606W with F814W STISon-NICMOS parallels, and better masking of moving target trails and the edges of chips.

Even in the version 2 images, there are still a few imperfections. A number of bright point sources had their central pixels "zeroed" due to being saturated (bleeding columns are also zeroed) or due to problems during CR removal. Also, there are typically a few hundred

pixels per field that are negative and which got through the DRIZ_CR process because they were in the median frames. The median frames used MINMAX rejection with NHIGH=2, $\mathrm{NKEEP}=1$. To fix this, the blotting and DRIZ_CR steps would need to be re-done. There are a number of frames for each field which have noticeable "X" patterns in them due to scattered light. There are at least two images without the "X" for each field, so it might be possible to remove this effect. Any updates to the status of the images and catalogs will be posted on the Space Telescope Science Institute's HDF-S web page which is currently at http://www.stsci.edu/ftp/science/hdfsouth/hdfs.html.

\section{STIS Data Reduction and Image Combination}

The STIS flanking field images, including the deep STIS-on-NICMOS image set, were reduced and DRIZZLE-combined in very much the same manner as the HDF-S STIS 50CCD deep field. The reader is referred to Section 3 of Gardner et al. (2000) for a full description. In this section, we describe the general process and document any differences from the deep field.

\subsection{Data Reduction}

Standard CCD image reductions were applied using the STSDAS CALSTIS pipeline and calibration images. However, the bias and dark subtractions made use of custom-created 
"super" versions of the calibration images. The standard CALSTIS pixel-to-pixel flatfield was used, but a standard low order flat was not available at the time of these calibrations so one was derived making use of the HDF-S images.

An additional custom calibration was made to account for "amplifier-ringing", variable horizontal artifacts induced by highly saturated pixels (see Section 3.4, Gardner et al. 2000). And finally, the sky background was measured and subtracted from each image.

\subsection{Image Combination}

We followed the standard DRIZZLE procedure using the STSDAS DITHER package tools (Fruchter et al. 1997) to combine the set of dithered images for each field. Starting with an initial estimate of the image-to-image offsets, a median image was created and used to identify and mask residual bad pixels and cosmic rays in the individual images. The final $\mathrm{x}, \mathrm{y}$ offsets were then measured by image-to-image cross-correlations.

The first iteration of the image-to-image $\mathrm{x}, \mathrm{y}$ offsets were as measured from the images' jitter files. As there were no differences in the commanded rolls to the spacecraft between the dithered flanking field exposures, the only rotation applied in the combining process was $\mathrm{a}+5.4$ degree rotation to North (5.55 degrees for the STIS-on-NICMOS field).

In the final image combination, the input images were both weighted and masked. The weighting maps are the inverse of the variance in the data as derived from the measured global noise characteristics (sky, dark counts, readnoise) of the data. For the deep field, the image weighting as input to DRIZZLE was scaled to account for the change in pixel size in the output image. We applied this scaling post-facto to the DRIZZLE-combined weight image (i.e. the version 1 weight image from the November 1998 release of these data) in combination with a scaling that forced the variance as predicted in $4 \times 4$ pixel regions of the final weight image to match the variance as actually measured from the same size regions of the background in the final combined object image. Because of the correlation between neighboring pixels in a drizzled image, the RMS noise measured from the final weight image is dependent on the size of the region being measured. Refer to Sections 3.5 and 4.1 of Casertano et al. (2000) and to Fruchter \& Hook (2002) for detailed discussions of this issue.

The mask for each input image was a combination of two pixel masks. One was the cosmic ray and residual bad pixel map determined in the DRIZZLE iterations. The second was a "static" mask (pixels "always bad" during this epoch of exposures), being itself a combination of custom-identified, persistent high sigma, deviant (hot and cold) pixels (Gardner et al. 2000), and a map of the unexposed detector border. 
The final DRIZZLE drop-size was a PIXFRAC of 0.8 ( 0.6 for the better sampled STISon-NICMOS field). Table 2 shows the RA and DEC of the center of each combined flanking field image plus the relevant "exposure log" information. Figures 6-8 show the final combined images.

\subsection{Photometric zeropoints}

We used a zeropoint of 26.386 for STIS (50CCD CLEAR) for infinite aperture in the ABMAG system. The zeropoint on the VEGAMAG system is 26.13. For the two-orbit STIS flanking fields, we estimate a limiting magnitude of 28.2 (AB mag $5 \sigma$ in an aperture of area 0.2 square arcsecond.) For the unconvolved STIS-on-NICMOS image, we estimate a limiting magnitude of 29.1 (AB mag $5 \sigma$ in a 0.2 square arcsecond aperture.)

\subsection{Current Status and Future Plans}

When the version 1 STIS FF images were first made available, we had not checked the astrometry. Therefore, the World Coordinate System in the headers was only accurate to the astrometry of the Guide Star Catalog, i.e., of order 0.5-1.0 arcsecond. For the version 2 release, these values have been updated to much better accuracy for the purposes of cataloging and for providing better positions for those observers doing follow-up work in small apertures and where high precision is required. The accuracy is now on the order of 0.1 arcsecond.

\section{NICMOS Data Reduction}

The data reduction steps for the NICMOS Flanking Fields were basically identical to those applied to the HDF-S NICMOS deep field. We describe the basic process here.

\subsection{Data Reduction}

Instrumental signatures were removed from each of the raw HST pipeline images in a series of steps, some of which made use of both the STSDAS pipeline software and standard calibration files, while other steps used customized software. First, the STSDAS task CALNICA was used to perform all of the pipeline's standard reductions except flatfielding and 
cosmic ray removal. Then, a customized removal of bias drifts and jumps was performed. This was followed by another run of CALNICA to perform cosmic ray removal and convert counts to count rate. Next, the sky and the "pedestal" biases were subtracted, and finally, the standard pipeline flat field was applied.

\subsection{Image Combination}

For each field, the calibrated images were drizzled into a single final image. Starting with the image-to-image dither offsets determined from the spacecraft jitter data, the images were combined and used to identify residual bad pixels and cosmic rays, which were then masked to aid in improved measures of the image offsets as derived from image to image cross-correlation. The improved measures of the offsets were used in repeated iterations of this process until the change in each offset was less than approximately 0.1 pixel.

The cosmic-ray rejection step followed that described above for WFPC2, with the exception that the rejection threshold varied across the field based on an error map. At this stage, one final instrumental artifact correction was made. Making use of an image combined using these final offsets, the so-called "Mr. Staypuft" (Bergeron et al. 2002) image persistence/ghosting was removed from the individual image for each dither position. These corrected images were used to produce the final DRIZZLE-combined image for each field. We used a DRIZZLE PIXFRAC of 0.7. We also employed the NIC-3 camera's image distortion corrections and used a DRIZZLE SCALE factor of 0.36974 to yield final pixels of 0.075 arcseconds. In this final image combination, the input images were both weighted and masked. The mask for each input image was a combination of two pixel masks. One was the residual cosmic ray and bad pixel map determined in the DRIZZLE iterations. The second was a "static" mask of pixels identified as having a persistent high sigma deviation during the epoch of these observations.

The pixel-to-pixel weighting maps are the inverse of the variance in the data as derived from the measured noise characteristics (sky, flatfield, gain, readnoise, etc.) of the data. The NICMOS deep field individual input image weighting maps were scaled to account for the change in pixel size in the output image. We applied this scaling post-facto to the DRIZZLEcombined weight image in combination with a scaling that forced the variance as predicted in $4 \times 4$ pixel regions of the final weight image to match the variance as actually measured from the same size regions of the background in the final combined object image.

Table 3 lists all the NICMOS NIC3 field coordinates and other relevant parameters. Figures 9-11 show NICMOS (NIC3) FFs 1-9 and the deeper F110W and F160W parallels 
done during the STIS-ON-NICMOS observations. Unlike all other HDF-S fields, the final combined NICMOS FF images were not rotated to have North up. Thus, those images are oriented at the original position angle of the observations, -174.64 degrees (-174.48 degrees for the deeper J and H-band STIS-on-NICMOS parallel images). Note, however, that the postscript versions of the NICMOS images shown here in Figures 9-11 were properly rotated to have North up and East to the left, and they are also properly oriented with North up and East to the left in the large mosaic that is shown as Figure 5 in Williams et al. (2000).

\subsection{Photometric zeropoints}

The most recent values for the zero points for NICMOS for infinite aperture in the ABMAG system are (F110W) 22.855 and (F160W) 22.865. The corresponding VEGAMAG zeropoints are 22.093 and 21.511, respectively. In the case of NICMOS, as opposed to WFPC2 and STIS, corrections from the standard-star aperture photometric measurements to "infinite aperture" were made using Tiny-Tim model point-spread functions (Dickinson et al. 2002). For the 2-orbit NICMOS F160W fields, we estimate a limiting magnitude (AB mag $5 \sigma$ in an aperture of area 0.2 square arcsecond) of 26.3. Similarly, we estimate limiting magnitudes of 26.9 for the F110W STIS-ON-NICMOS parallels, and 26.7 for the F160W STIS-ON-NICMOS parallels.

\section{Astrometry}

In order to verify the quality of the final astrometric information in both image headers and catalogs, several cross checks have been made. The flanking field images for all three instruments, along with the deep fields themselves and the STIS-on-NICMOS data were all drizzled onto a single large mosaic image with a scale of $0.15^{\prime \prime}$ per pixel. The information for mapping from the input images to the mosaic was taken from the image header World Coordinate Systems (WCS). The catalogs, which also have their astrometry derived from the same WCS, were then over-plotted and the match inspected carefully by eye. Some problems with the initial catalogs were identified and corrected. This effectively ruled out gross problems with the match between the catalogs and the image WCS information, but was a purely internal check.

The next stage was the verification that the catalogs were consistent where there were overlaps between different flanking field exposures and different cameras. Suitable stars were identified in overlap regions and the positions given in the different catalogs were compared. 
Finally, the positions of six USNO astrometric reference stars from the Zacharias et al. (1998) catalog were compared to the positions in the catalogs from the images. In all cases, both the internal and external astrometric errors were found to be of the order of 50 milliarcseconds (mas), with the largest errors, up to 100 mas, found for the NICMOS flanking fields, which have the largest pixel scale and were the most difficult to calibrate astrometrically.

\subsection{WFPC2 Astrometry and Large Mosaics}

The headers of the WFPC2 frames contain approximate information about the pointing, orientation, and scale of the images which is deduced from observatory engineering information and the positions of the guide stars. The pointing of the telescope is locked into the Guide Star Catalog reference frame and is typically accurate to no better than $1^{\prime \prime}$. This information is stored as a linear representation in the World Coordinate section of the image headers (WCS). The drizzling of the individual flanking field mosaic images removed the geometric distortion present in the original frames and positioned the four chips relative to each other on the sky with an accuracy of a few tens of milliarcseconds. In this process, the original World Coordinate System information was also transferred. For the purposes of cataloging as well as the automatic preparation of multiple-field mosaics, a much higher accuracy was desirable and it was also intended to present positions in the Hipparcos (ICRS) reference frame. Fortunately, astrometric imaging of the HDF-S field was performed by the US Naval Observatory (Zacharias et al. 1998) and a list of star positions covering the entire flanking field region was available. The number of such useful reference points varied from field to field but was typically 4, with the worst being FF6 (only one) and the best FF8 (8). Some of the astrometric objects were extended, double, or saturated in the WFPC2 frames and were not used.

The pixel positions of these reference objects were measured using the IMEXAMINE task in IRAF. These were then compared to the true celestial positions using the CCMAP task (with the FITGEOMETRY = RSCALE setting) in the IMCOORD package in IRAF. The WCS in the image headers was adjusted accordingly, with a listing of the new WCS keyword values being used to create a script to update the headers of both the science and the weight images. The fit which was performed allowed for a shift, a rotation, and a change of scale. The RMS residuals of such fits were 20-30 mas in most cases. When the intrinsic errors of the USNO catalog are considered, along with other possible sources of error, the likely absolute astrometric accuracy for the WFPC2 WCSs, and hence the catalogs derived from them, is of order 50mas. Unlike the case of the smaller and mostly non-overlapping STIS and NICMOS flanking field images, there was no attempt to use more stars from the 
CTIO Big Throughput Camera (BTC) wide field image of Teplitz et al. (1998) with the USNO catalog reference frame applied to improve the astrometric solution further for the WFPC2 flanking field images. The updated image headers with better astrometry simply based on the reference objects from the USNO catalog within each WFPC2 flanking field as described above were used to provide the positions in the source catalogs. The 9 contiguous flanking field images, along with the F814W mosaic from the deep field, were then mosaiced into a single $6000 \times 6000$ pixel image with a scale of 0.1 arcsecond per pixel using an enhanced version of the DRIZZLE (V1.4) task which derived the mapping from input to output pixel positions from the image WCS. The result is shown as Figure 12 and is available via the STScI HDF-S WWW pages. In addition to this, as mentioned earlier in Sections 2 and 3.2, an even larger mosaic including all of the flanking fields in all instruments plus the three deep fields (STIS, WFPC2, and NICMOS) was created and is shown in Figure 5 of Williams et al. (2000).

\subsection{STIS Astrometry}

Where possible, positions of reference stars from the USNO astrometric catalog were used to correct the positions and WCS information in the data headers of the STIS FF images. This required a reference star in that individual FF, which was not available for all fields. Where there was no reference star directly from the USNO catalog, we have used the CTIO Big Throughput Camera (BTC) wide field image of the area (Teplitz et al. 1998) to provide more stars to cover all of the STIS fields. We have used the USNO reference frame to update that of the BTC image, and have transferred it hence to the STIS FF images where necessary.

Specifically, the final STIS astrometry was obtained by summing all of the BTC camera images in 5 bands and using the WCS from it as reference. Typically, 3 or 4 reference points in each frame were used, and yielded an internal RMS of 40 mas or better. (In many cases one point was rejected but the final fits always came from at least 3 stars.) These fits allowed scale change, rotation, and shifts (with the scale the same in both directions). The results had slightly varying scales and small rotations of a few hundredths of a degree, centered close to 0 . We imposed a constant scale of 25 mas per pixel and aligned North in the $+y$ direction. The reference pixel was set to the centers of the images, as well. After drizzling the resultant images onto the BTC WCS, the two were blinked for comparison. Typical errors were of order 0.1 BTC pixels (40 mas).

The astrometric World Coordinate System (WCS) for the final image of each field was adjusted so that positions of objects in the STIS images match those in the BTC image. 
In turn, the BTC coordinate system had been set so that object positions matched those determined in a special astrometric catalog of the HDF-S field compiled by the USNO. Using the BTC WCS, several stars, at least 3 for each image, were used as reference points and fits were made with $\mathrm{x}, \mathrm{y}$ shifts, rotation, and plate scale as free parameters. Shifts of order $1.3^{\prime \prime}$ were needed and the changes were made to the RA and Dec assigned to the fiducial reference pixels. Changes to the rotations (already performed to set North to $+y$ in each image) and plate scale (0.025 arcsecond per pixel) were not needed. The combined residuals of these fits to the BTC and those of the BTC with respect to the USNO astrograph object positions are approximately less than or equal to $0.1^{\prime \prime}$.

\subsection{NICMOS Astrometry}

The astrometry of the NICMOS flanking field images was improved in a similar way to the STIS images. In this case, there were no USNO astrometric stars which could be used directly and the BTC I-band image was used as reference. Objects were identified on the BTC image which could also be seen in the NICMOS images, their positions deduced from the WCS in the header of the BTC image, and an astrometric solution performed. The WCS in the NICMOS frame was adjusted accordingly, with the scale constrained to be 0.075 arcsecond per pixel. The internal errors in the astrometric fit, typically to 3 or 4 objects, were of order 40 mas. However, the large pixels of the NICMOS and BTC images, and inevitable difficulties in matching objects at significantly different wavelengths, combined with the probable residual errors in the BTC WCS, mean that the final absolute accuracy of the NICMOS flanking field WCS is estimated to be of order $0.15^{\prime \prime}$.

\section{Source Detection and Catalogs}

Average values for the estimates of limiting magnitude per camera and type of flanking field have been given in earlier sections. Estimates of limiting magnitude are directly related to source detection and catalogs, thus we present this information here in Table 4 which gives a summary of this information as well as an estimate of the $5 \sigma$ limiting magnitude for each individual flanking field in each of the three cameras, as measured in an aperture of 0.2 square arcseconds in area. To determine a practical limiting magnitude, we extracted from each flanking field catalog the non-border objects whose flux in a 0.2 square arcsecond aperture was 5 times $( \pm 5 \%)$ the uncertainty in the flux measurement, i.e., between 4.95 and 5.05 times the SExtractor quoted uncertainty. We then found the mean magnitude for those objects. 
Objects in the flanking fields were identified and characterized using the Source Extraction (SExtractor) package (Bertin \& Arnouts 1996), version 2.1.0. Our use of this software was very similar to the process described in detail by Casertano et al. (2000). For expanded descriptions, please refer to Section 5 of that paper while we restrict these notes to issues and parameter settings particular to the flanking field data. Because of possible effects on the flux (and magnitudes) and uncertainties recorded in the catalogs, it should be recalled here that since flux in adjacent pixels in the output image can come from the same pixel in the input images, the noise is strongly correlated in the final drizzled images. Also, though the outer noisiest borders around all the images were masked before cataloging, some lowweight pixels around the edge of the WFPC2 and STIS images still had residual cosmic rays which may have affected the cataloging. Thus, all objects in the catalogs originating from the borders of the images were flagged (IMAFLAG=1) for use with caution.

The SExtractor object identification and measurement process was conducted in a virtually identical manner for all of the flanking fields. The following process was repeated for each field. Three images were input to SExtractor: an edge-masked object image, an rms image, and a flag image. For WFPC2 and STIS, the flanking field images have noisy border areas with cosmic ray residuals due to partial overlap of the input images in those regions. We masked these borders, and portions of the interchip WFPC2 regions, according to which pixels in the weight image were below threshold values corresponding to a minimum number (2-3) of overlapping input images. We then created a flag image in which the regions of the image where all of the input images overlapped were set to 0 and the less than full $\mathrm{S} / \mathrm{N}$ regions (basically, additional border area not trimmed by the masking) were set to 1 . SExtractor recorded this flag image value in the IMAFLAGs parameters for each object in the catalog, thus flagging each "border" object. The rms image is simply 1 divided by the square root of the weight image. We used this rms image as SExtractor's "WEIGHT_MAP" to force the software to use our spatially dependent drizzled weights (which, e.g., accounted for variation in exposure time per pixel) in adjusting the detection threshold which SExtractor applied to each pixel. Also, SExtractor used our RMS map rather than its own internal estimates to assign the error uncertainties to its flux and magnitude measurements.

We used the defaults for SExtractor's object identification parameters (SExtractor parameters are identified by capitalized words in the following description) except as specified in this description and listed for each instrument's set of fields in Table 5. Some of the input parameters naturally varied between the three instruments, e.g. PIXEL_SCALE. The detection threshold settings varied due to differing noise characteristics in the combined images caused by instrumental differences as well as calibration and data processing differences.

SExtractor first measured and subtracted a background from the image. The back- 
ground value assigned to each pixel was calculated in two steps. First, the image was divided up into a grid of boxes, with the size of each box set by the parameter BACK_SIZE. A mean pixel value was measured in each box. Second, this background map was smoothed by a boxcar filter of BACK_FILTERSIZE, and the resulting pixel-to-pixel background values were subtracted from the input image. SExtractor then optimized object detection by convolving the image with a filter kernel. For this filter, we input a Gaussian whose half-width corresponded to the approximate point source FWHM. SExtractor then identified objects in the filtered image as being any group of 16 or more connected pixels, each pixel having a value greater than our chosen $\sigma$ detection threshold. SExtractor then "deblended" any subcomponents of these connected pixels into separate objects in a process controlled by the DEBLEND_MINimum contrast and DEBLEND_NTHRESHolds parameters. Before being accepted, these object identifications had to pass one further test, SExtractor's "cleaning" process. The wings of each object were extrapolated using a Moffat profile scaled by the CLEANing parameter. Faint "neighboring" and/or temporarily "deblended" objects could then be merged or removed from the catalog due to this re-assignment of flux to the wings of a brighter neighbor.

We iterated on the above process using SExtractor's "check" images to first test the background fitting parameters and then to choose the best object identification as we varied the DETECTION and DEBLEND_MIN parameters. Once object identification was complete, SExtractor performed the shape and photometric measurements using the pixel values from the original input object image. It first subtracted a background from each pixel which we chose to be the "local" background measured within an annulus around each object whose width was set by BACKPHOTO_THICK. SExtractor also retested which pixels to measure with an "analysis" threshold for which we always used the same value as our detection threshold. Finally, SExtractor attempted to classify the objects as point-like or extended, i.e. stars or galaxies, and gradations between, making use of the SEEING_FWHM which we input, and a "neural-network" weighting. We used SExtractor's default neural network.

For each bandpass of the deep "STIS-on-NICMOS" WFPC2 and NICMOS flanking fields, we used SExtractor in two-image mode. First, the object identification phase was performed on an input image which we created from the inverse variance weighted sum of the images from the two bandpasses. Also, we used an RMS image which was derived from the sum of the two individual weight images. The object pixel locations identified in the detection image were the pixel locations that SExtractor extracted from the individual bandpass images when it performed the photometric measurements as cataloged for each bandpass. The shape measurements, half-light radius, object classification, and anomaly flags cataloged for these objects are as measured from the detection image. 
A number of objects which were clearly spurious detections were deleted from the catalogs. In the WFPC2 FFs, these were all related to diffraction spikes. In the STIS FFs, they were bright object ghosts and scattered light as well as diffraction spike artifacts. In the NICMOS FFs, these were diffraction spikes and, in NIC FF3, numerous residual cosmic-rays along the image borders. However, except for the NICMOS FF3 border artifacts, only the neighbors of objects bright enough to produce diffraction spikes were checked.

There remain a small number of misidentifications in the catalogs which no straightforward adjustment of SExtractor's object identification parameters could overcome. These problematic ID's fall into three categories. Patchy extended objects such as faint, face-on spiral galaxies occasionally have their brighter subsections cataloged as separate objects. A few separate but closely neighboring objects are identified as single objects. These are sometimes close pairs of stars, and sometimes closely neighboring, interacting, or superimposed galaxies. And third, there are a few CR artifact objects in the STIS and WFPC2 border regions. (Though only a few may be residual CR's, all objects near the borders were flagged, as the photometry of large extended objects which may not be entirely in the field of view should be treated with caution.) Finally, the photometry of bright point sources in the WFPC2 FFs should be treated with caution due to the occasional object with "zeroed" peak pixels.

\subsection{The Catalogs}

For convenience, we created a merged catalog, per instrument, of all the individual flanking field catalogs. In Table 6, we present a small subset of the catalog parameters and photometry for a small representative sample from the merged WFPC2 catalog. Table 7 and Table 8 contain small subsets of the same data from the merged STIS and NICMOS catalogs, respectively. The extended catalogs contain the full set of our SExtractor measurements such as the elliptical shape parameters and aperture photometry in aperture diameters from $0.0875^{\prime \prime}$ to $4.0^{\prime \prime}$.

The WFPC2 flanking fields overlap one another, and there are flanking fields which overlap the main (deep) HDF-S fields, thus the same object may appear in more than one of the individual fields' catalogs. In the merged catalogs, we accounted for these objects by providing a column, DUP, which identifies these objects as well as ranking them by $\mathrm{S} / \mathrm{N}$. This parameter was left blank for the objects in both the WFPC2 and NICMOS deep (parallel to STIS-on-NICMOS) flanking fields, though there are catalog measures of most of these objects in two bandpasses. There are a few flanking fields from one instrument that overlapped flanking fields from one of the other instruments: STIS FF2 with WFPC2 FFs 
3, 4, and 8, STIS FF3 with WFPC2 FF8, STIS FF9 with NICMOS FF7, and NICMOS FF1 with WFPC2 FF6. Though objects may appear more than once among the online catalogs for these individual fields, they are not identified in the merged catalog DUP parameter. Except for DUP, in the merged catalogs blank entries indicate that SExtractor was unable to produce a sensible measurement, e.g. values of $\mathrm{M}<0$ or $\mathrm{M}>40$ or $\sigma_{M}>2$ from the individual catalogs are left blank in the merged catalogs.

The catalog parameters which we provide in each of Tables 6,7 , and 8 are:

Field: An instrument identifier and field number from which the associated line of measurements was made.

Dup: In merging the catalogs, we first sorted by Right Ascension and then by Declination. Some WFPC2 flanking fields overlap each other and some of the flanking fields from each of the instruments overlap HDF-S deep fields. For the fields which overlap each other, we identify objects which were measured in more than one flanking field, as well as identifying objects which were also measured in any of the HDF-S deep fields. The DUP column in the catalogs flags these duplicate measures. A flag value of 1 indicates that that particular listing has a duplicate measurement in another flanking field but that this measurement has the highest $\mathrm{S} / \mathrm{N}$. A value of 2 indicates that there is a duplicate measure in another flanking field and this measurement is of lower $\mathrm{S} / \mathrm{N}$. A value of 3 indicates that the object is also found in one of the HDF-S main field catalogs. This flag is left set to 0 for all catalog entries from the multiple bandpass exposures of the deep WFPC2 (parallel to STIS-on-NICMOS) flanking field.

ID: The SExtractor identification number. The objects in the list have been sorted by Right Ascension (first) and Declination (second), and thus are no longer in catalog order.

HDFS J Catalog ID: The minutes and seconds of Right Ascension and Declination, from which can be constructed the catalog name of each object. To these must be added 22 hours (RA) and -60 degrees (Dec). For example, the first object shown in the sample WFPC2 catalog (Table 6) is HDFS_J223242.47-603505.7, at RA $22^{h} 32^{m} 42.47^{s}$, Dec $-60^{\circ}$ $35^{\prime}$ 05.7", Equinox J2000. Note that in the full, on-line catalogs, the full catalog object ID name including hours of Right Ascension and degrees of Declination is given.

$\mathbf{x}, \mathbf{y}$ : The $\mathrm{x}$ and $\mathrm{y}$ pixel positions of the object on the images.

$m_{I}, \sigma\left(m_{I}\right), m_{a}$ : The isophotal magnitude in the specified bandpass, in this case, for example, the F814W image $\left(m_{I}\right)$, its uncertainty, and the "MAG_AUTO" $\left(m_{a}\right)$ magnitudes. The magnitudes are given in the AB system (see Oke 1971), where $m=-2.5 \log f_{\nu}-48.60$ with $f_{\nu}$ in $\operatorname{erg} \mathrm{cm}^{-2} \mathrm{~s}^{-1} \mathrm{~Hz}^{-1}$. The isophotal magnitude was determined from the sum of 
the counts within the detection isophote, set to be $0.5 \sigma$. The MAG_AUTO is an elliptical magnitude (see Kron 1980) determined from the sum of the counts in an elliptical aperture. The semi-major axis of this aperture was defined by 2.5 times the first moments of the flux distribution within an ellipse roughly twice the isophotal radius. However, if the aperture defined this way would have a semi-major axis smaller than 3.5 pixels, a 3.5 pixel value was used.

$r_{h}$ : The half-light radius of the object in the detection image, given in milliarcseconds. The half-light radius was determined by SExtractor to be the radius at which a circular aperture contained half of the flux in the MAG_AUTO elliptical aperture.

s/g: A star-galaxy classification parameter determined by a neural network within SExtractor, and based upon the morphology of the object in the detection images. (See Bertin \& Arnouts 1996 for a detailed description of the neural network.) Classifications near 1.0 are more like a point source, while classifications near 0.0 are more extended.

Flags: Flags are explained in the table notes, and include both the flags returned by SExtractor, and additional flags we added while constructing the catalog.

\section{Number counts}

The WFPC2, STIS, and NICMOS number counts were computed to demonstrate the relative depth of the flanking fields and for comparisons to other number count studies. Where a source was observed several times with the same entry, we included only the catalog entry with the highest signal-to-noise ratio. We also used the SExtractor CLASS parameter to reject star-like objects from our number count computations. A CLASS value of 0.9 was used as the dividing line, with anything greater than that being rejected.

\subsection{WFPC2}

The WFPC2 flanking fields comprise the most extensive and overlapping fields of any of the three instruments. In addition to 9 shallow fields of 2 orbits each in F814W, we also imaged a deeper 9-orbit single field with 4 orbits being dedicated to F606W and 5 orbits to $\mathrm{F} 814 \mathrm{~W}$. The I-band number counts for the shallow fields covering a non-overlapping area of $1.092 \times 10^{-2}$ degree $^{2}$ and the WFPC2 HDF-S deep flanking field (STIS-on-NICMOS parallel) counts are shown in Figure 13. We truncate the number counts at the faint end where incompleteness starts to become significant, which for the WFPC2 2-orbit FFs is at I_814 MAG_AUTO = 25, and for the 5-orbit F814W STIS-on-NICMOS WFPC2 parallel is 
at I_814 MAG_AUTO $=26$.

As can be seen in Figure 13, the number counts for the various WFPC2 flanking fields and deep field are similar out to the completeness limit. For comparison purposes, we also plot I-band number counts found from surveys by Hall (1984), Koo (1986), Tyson (1988), Lilly et al. (1991), and Gardner et al. (1996). The number counts of these various surveys agree with the number counts from the WFPC2 flanking fields.

\subsection{STIS}

With a few minor exceptions (mainly STIS FF2 with WFPC2 FFs 3, 4, and 8, as noted in Section 7.1, above) and one major one (the STIS-on-NICMOS image), most of the STIS FFs did not overlap significantly with any of the FFs imaged by any of the other instruments. Each STIS FF consisted of two orbits (5100 seconds total) of exposure time in 50CCD CLEAR mode. The STIS-on-NICMOS image consisted of 9 orbits (25,900 seconds total) of exposure time in 50CCD CLEAR mode.

The STIS 50CCD number counts for the unconvolved STIS-on-NICMOS image are shown to MAG_AUTO $=28.0$, and for the 9 combined 2-orbit STIS FFs, to MAG_AUTO $=27.0$. At fainter magnitudes, completeness corrections become significant. The number counts for the 9 combined STIS FFs are shown in Figure 14, along with the number counts for the unconvolved STIS-on-NICMOS image, and for the STIS deep field, which are shown for comparison.

\subsection{NICMOS}

The NICMOS flanking field imaging was done solely in F160W for FFs 1-9, for a duration of approximately two orbits each, in parallel with the primary WFPC2 FFs 1-9. In parallel with the primary 9-orbit STIS-on-NICMOS imaging, both F110W and F160W were used in alternating fashion, with equal amounts of time being spent in each filter. Only the NIC3 images were used for cataloging due to the poorly focused NIC1 and NIC2 images.

The NICMOS number counts were computed using a similar method to that used for the WFPC2 and STIS fields. The result is shown in Figure 15 for the NICMOS H(1.6 $\mu \mathrm{m})$ band. For the 9 combined 2-orbit NICMOS FFs, the H-band number counts are truncated at MAG_AUTO $=25.5$, and for the deeper STIS-on-NICMOS NICMOS parallel, the Hband number counts are truncated at MAG_AUTO $=26.5$, with the greater significance of incompleteness at magnitudes fainter than those being the reason for the truncation, as with 
the WFPC2 and STIS number counts. Also shown for comparison purposes in Figure 15 are H-band number counts for the NICMOS deep field of Fruchter et al. (2002).

\section{Conclusions}

We have presented the entire suite of HDF-South flanking field observations in all three instruments, describing the observations, and data reduction processes. We have reviewed updates to the astrometry and have described extended mosaics of multiple flanking fields in detail. We have also presented catalogs and number counts derived from them. The number counts are broadly consistent with those of previous surveys. The original driving force for the flanking fields was the need for more targets beyond the deep fields for long-slit spectroscopy at ground-based telescopes. In meeting this need, this large dataset is also useful for other purposes, especially studies of galaxy evolution involving number counts, sizes, and morphologies, as well as studies of potential absorbers along the lines of sight near the QSO. They may also be used as "baseline" images for later follow-up observations to hunt for distant supernovae. These data can also be useful for galactic studies such as star counts. While their utility has mainly been seen by us as involving the kinds of studies mentioned above, there are undoubtedly many other uses of which we have not yet conceived, and which may represent their greatest potential.

We thank our program coordinator at STScI, Andy Lubenow, for his work on implementing the program, the long range planners and the calendar builders who scheduled it, and all of the many people who contributed to all the various aspects of this project. This work was supported by grant GO-8058.01-96A from the Space Telescope Science Institute, which is operated by the Association of Universities for Research in Astronomy, Incorporated, under NASA contract NAS5-26555. 


\section{REFERENCES}

Baggett, S., et al. 2002, in HST WFPC2 Data Handbook, v. 4.0, ed. B. Mobasher, Baltimore, STScI

Bergeron, L. E., et al. 2002, NICMOS Instrument Science Report, in preparation

Bertin, E., \& Arnouts, S. 1996, A\&AS, 177, 393

Casertano, S., et al. 2000, AJ, 120, 2747

Cohen, J., et al. 2000, ApJ, 538, 29

Dickinson, M. E. 1995, in Galaxies in the Young Universe, ed. Hippelein et al., p. 144, Berlin: Springer

Dickinson, M. E., et al. 2002, in HST NICMOS Data Handbook, v. 5.0, ed. B. Mobasher, Baltimore, STScI

Dressler, A., Oemler, A., Sparks, W. B., \& Lucas, R. A. 1994, ApJ, 435, L23

Ferguson, H. C., Dickinson, M. E., \& Williams, R. E. 2000, ARA\&A, 38, 667

Fruchter, A. S., Hook, R. N., Busko, I. C., Mutchler, M. 1997, in The 1997 HST Calibration Workshop, Casertano, S., Jedrzejewski, R., Keyes, C. D., Stevens M., eds., 518 (Space Telescope Science Institute, Baltimore)

Fruchter, A. S., \& Hook, R. N. 1997, Proc. SPIE, 3164, 120

Fruchter, A. S., \& Hook, R. N. 2002, PASP, 114, 144

Fruchter, A. S., et al. 2002, AJ, in preparation

Gardner, J. P., et al. 1996, MNRAS, 282, L1

Gardner, J. P., et al. 2000, AJ, 119, 486

Griffiths, R. et al. in IAU Symposium 168: Examining the Big Bang and Diffuse Background Radiations, ed. M. Kafatos and Y. Kondo, Dordrecht: Kluwer

Hall, P., \& MacKay, C. D. 1984, MNRAS, 210, 979

Koekemoer, A. M., et al. 2002, "HST Dither Handbook", Version 2.0 (Baltimore: STScI)

Koo, D. 1986, ApJ, 311, 651 
Kron, R. G., 1980, ApJS, 43, 305

Lilly, S., Cowie, L., \& Gardner, J. P. 1991, ApJ, 369, 79

Oke, J. B. 1971, ApJ, 170, 193

Teplitz, H. I., et al. 1998, BAAS, 193, 75.07

Tyson, J. A. 1988, AJ, 96, 1

Williams, R. E., et al. 1996, AJ, 112, 1335

Williams, R. E., et al. 2000, AJ, 120, 2735

Zacharias, N., Corbin, T., Zacharias, M., Rafferty, T., Seidelmann, P. K., \& Gauss, F. S. 1998, BAAS, 193, 75.09 

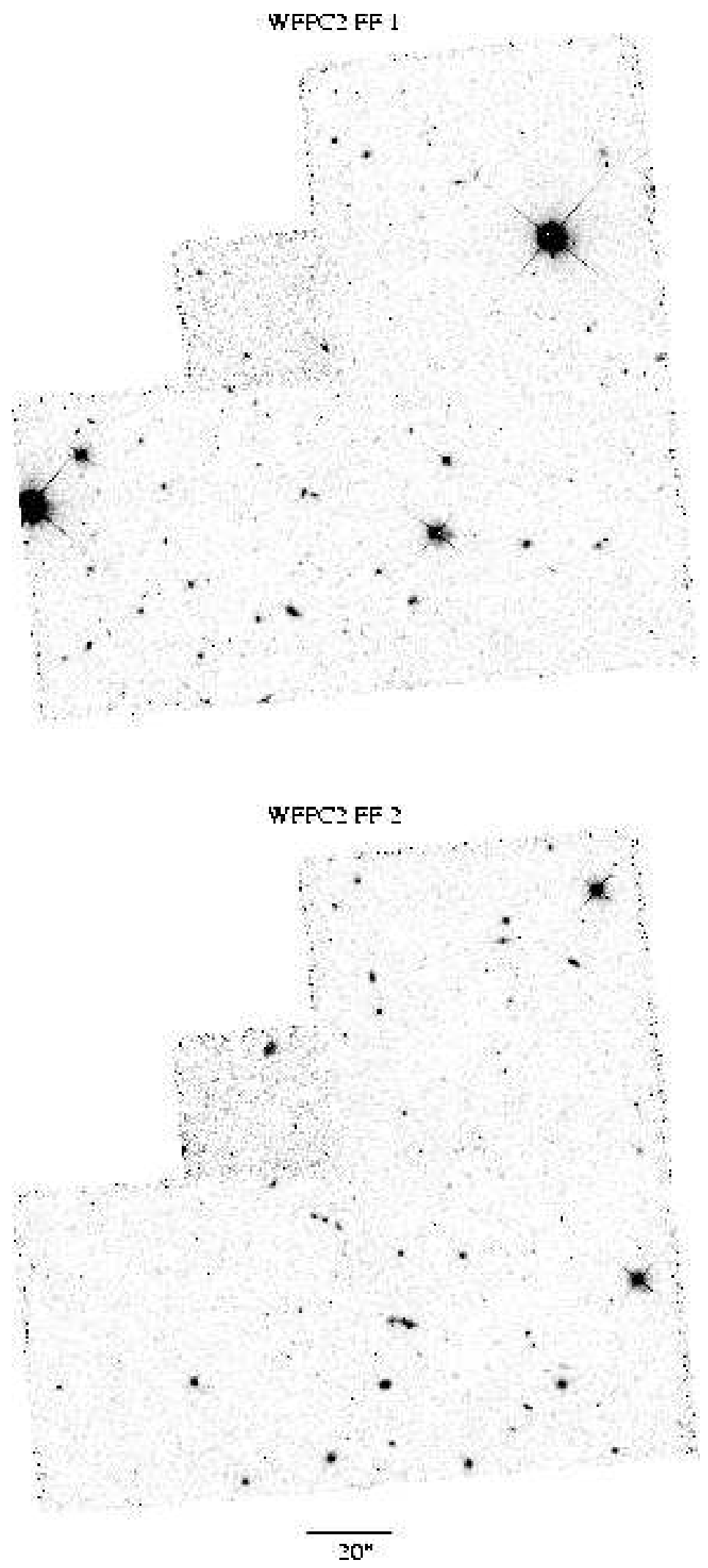

Fig. 1.- WFPC2 flanking fields 1-2. F814W, 5100s. North is up, East is to the left. 

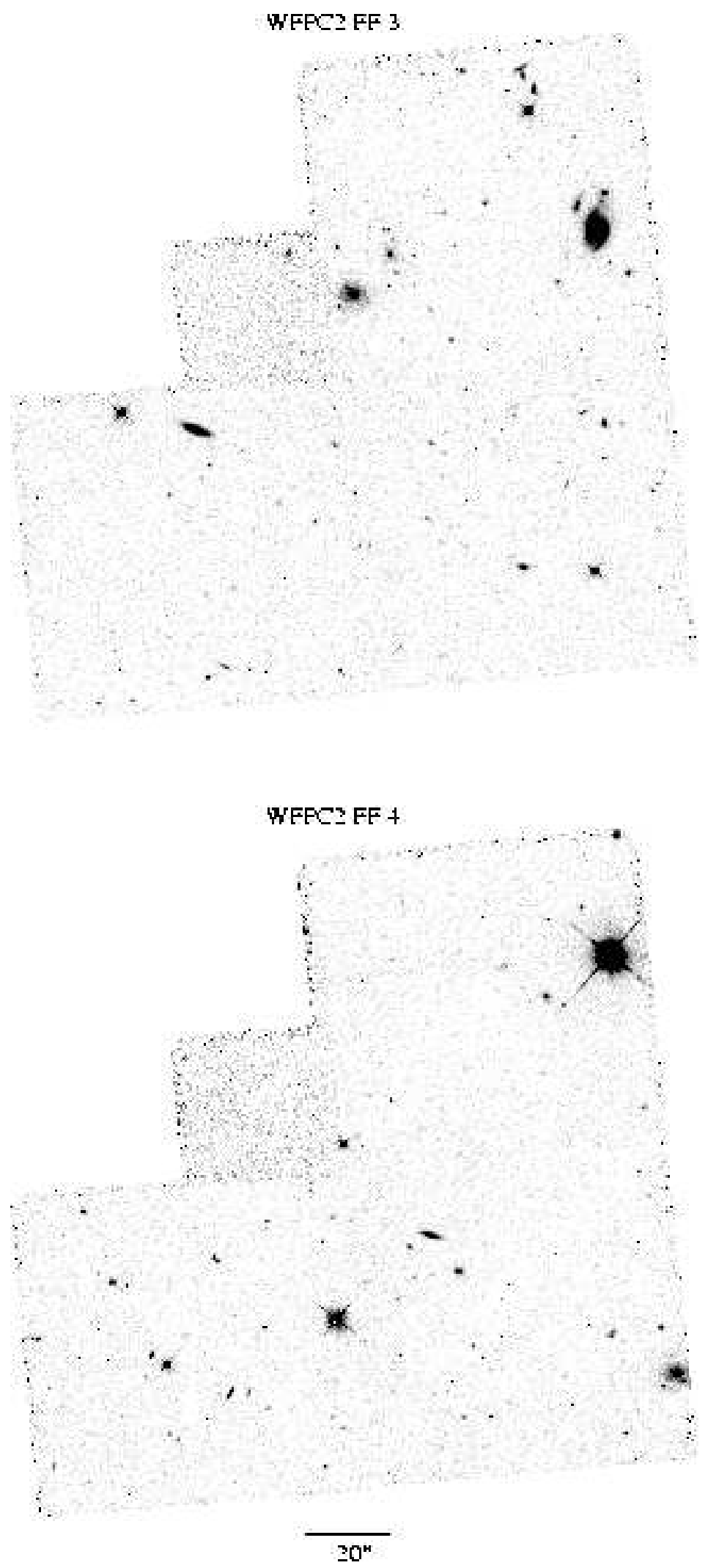

Fig. 2.- WFPC2 flanking fields 3-4. F814W, 5100s. North is up, East is to the left. 

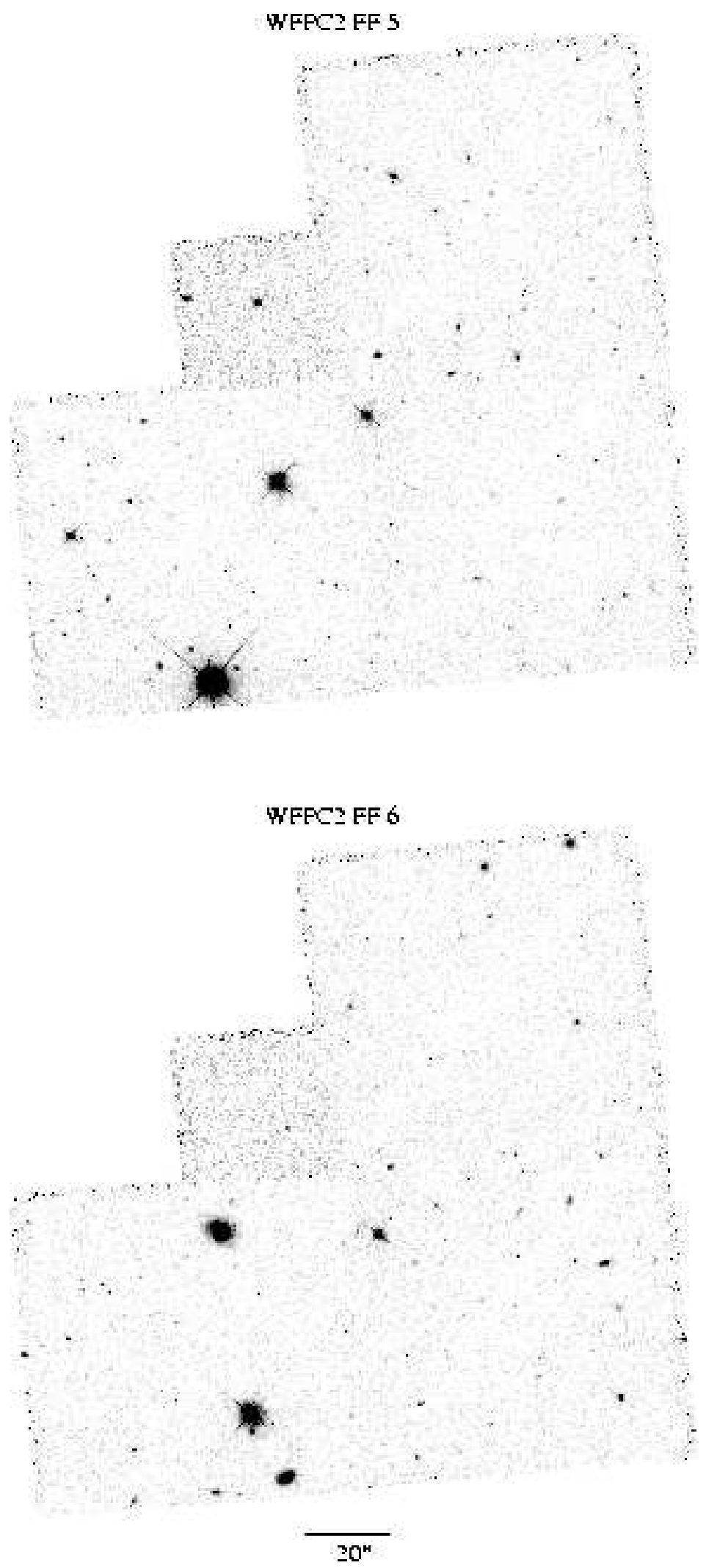

Fig. 3.- WFPC2 flanking fields 5-6. F814W, 5100s. North is up, East is to the left. 

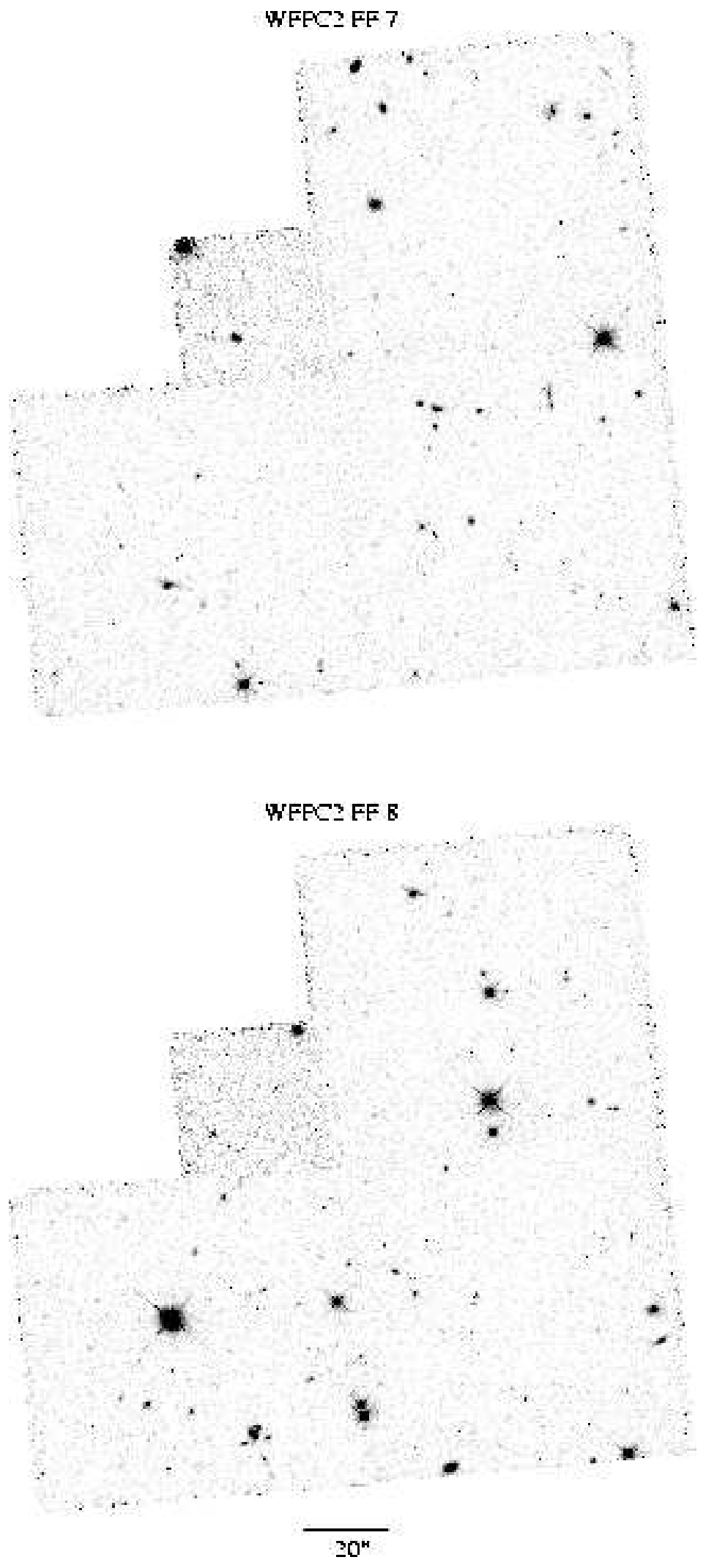

Fig. 4.- WFPC2 flanking fields 7-8. F814W, 5100s. North is up, East is to the left. 


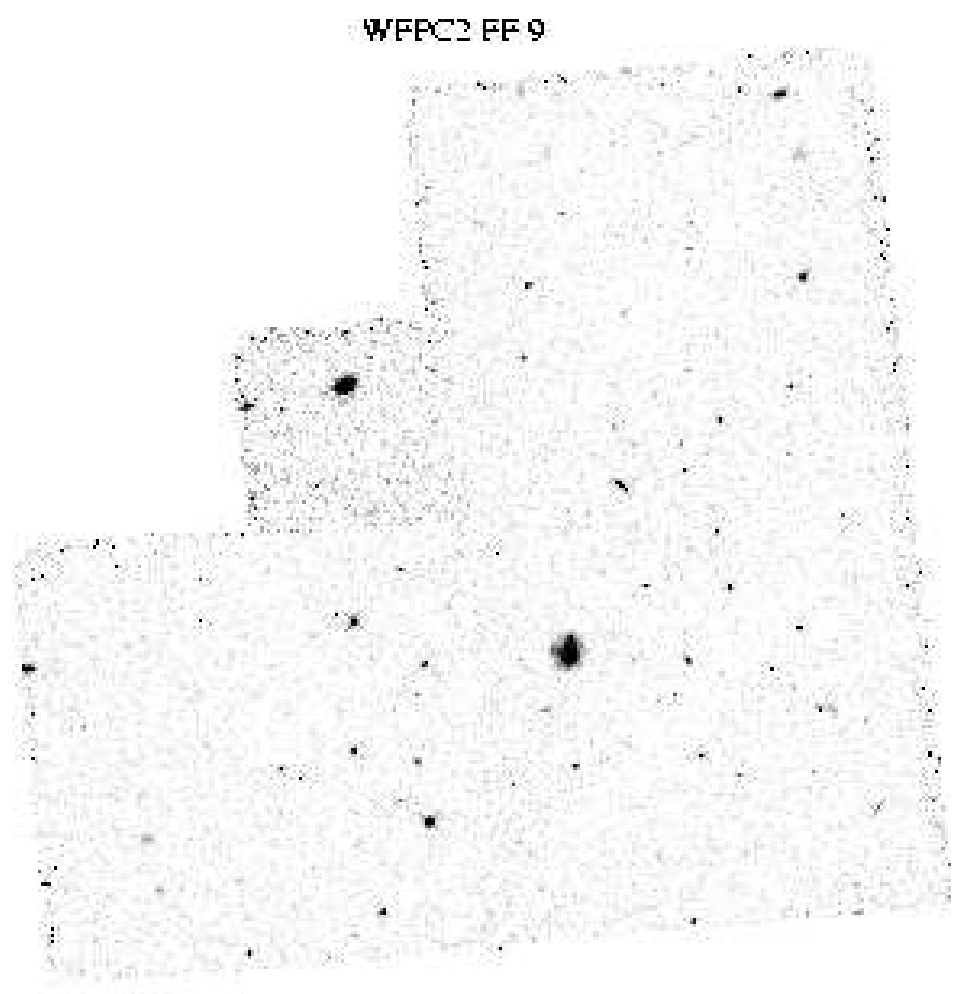

WFPC2 FF Deep F606w + Deep FB14W

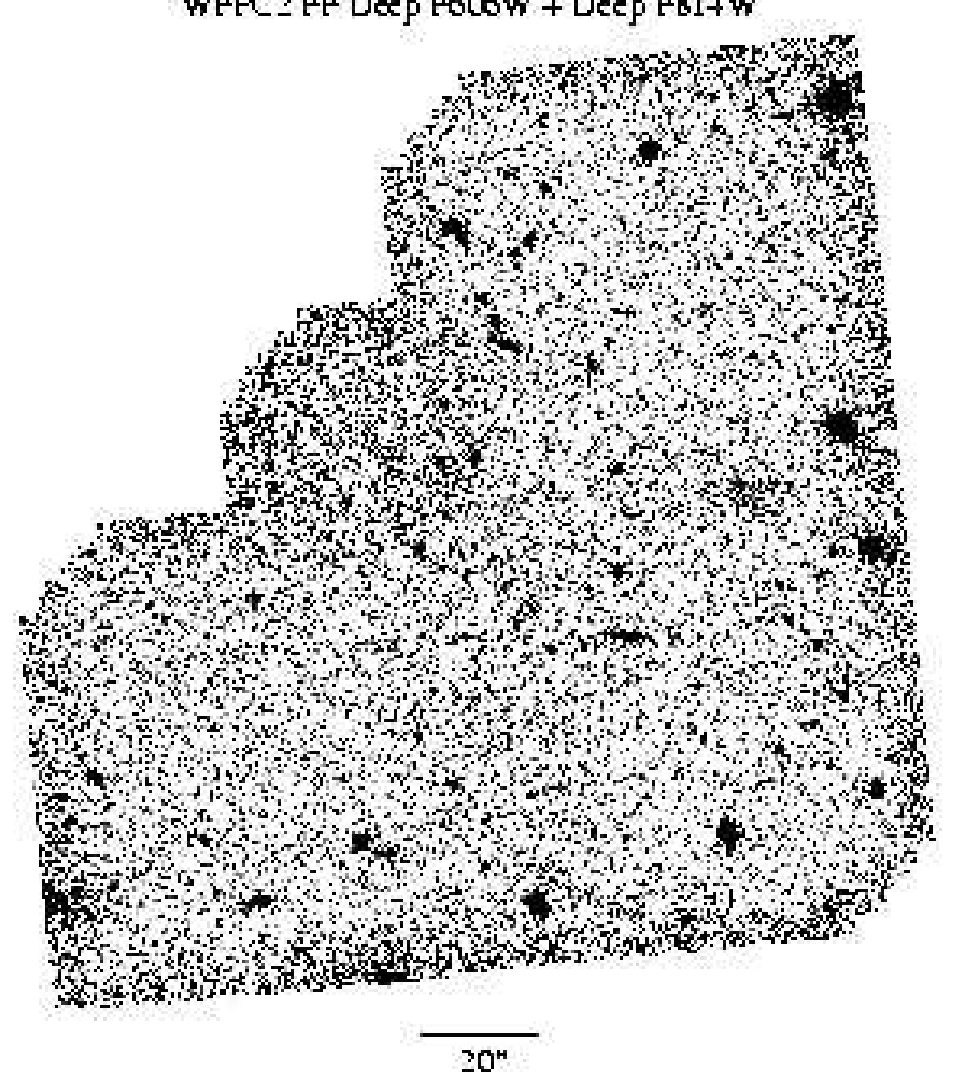

Fig. 5.- Top: WFPC2 flanking field 9. F814W, 5100s. Bottom: WFPC2 deep flanking field. F606W, 9400s, combined with F814W, 11,800s. Contrast stretched to point out low surface brightness object at center right. North is up, East is to the left. 

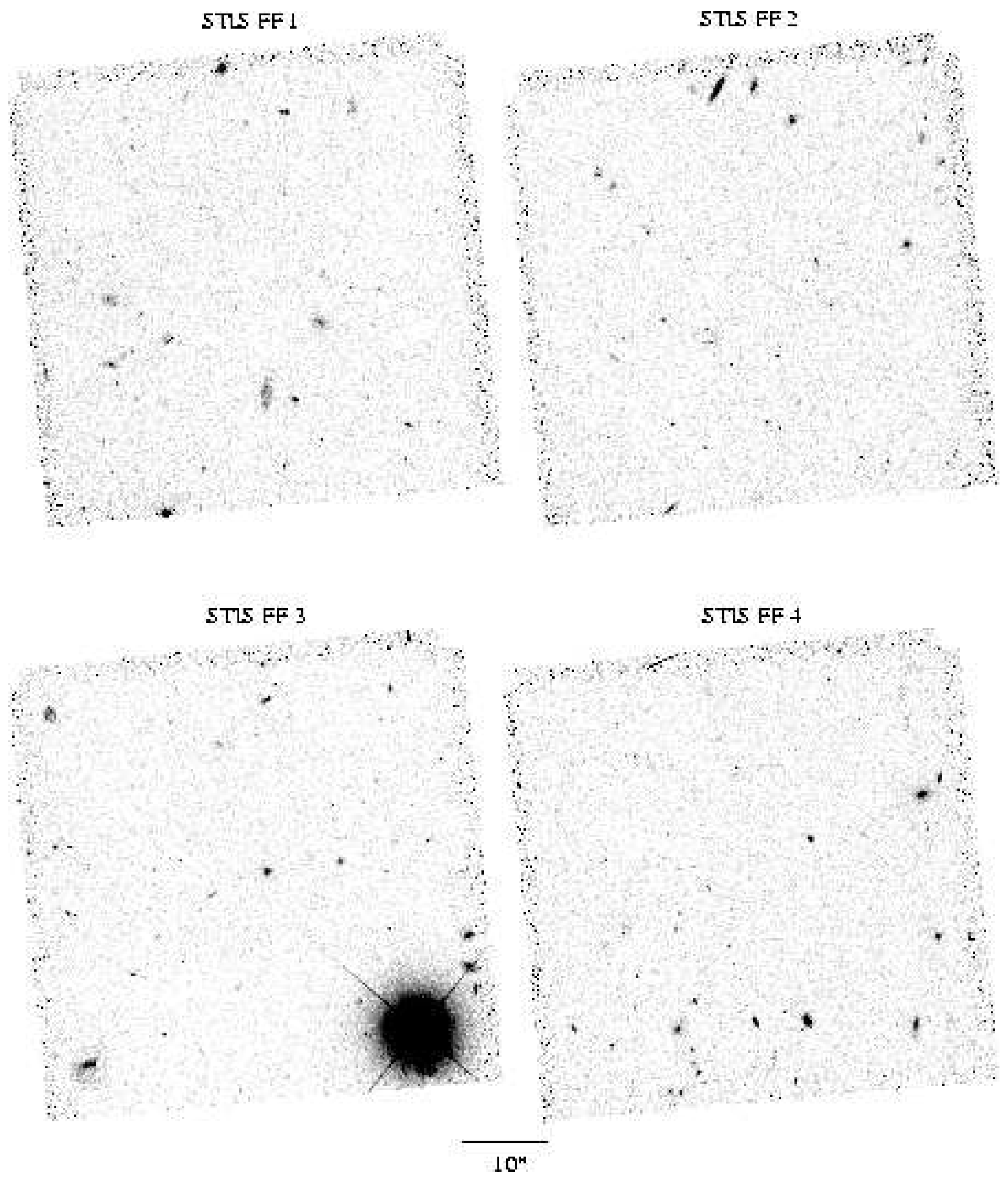

Fig. 6.- STIS flanking fields 1-4. 50CCD, 5100s. North is up, East is to the left. 

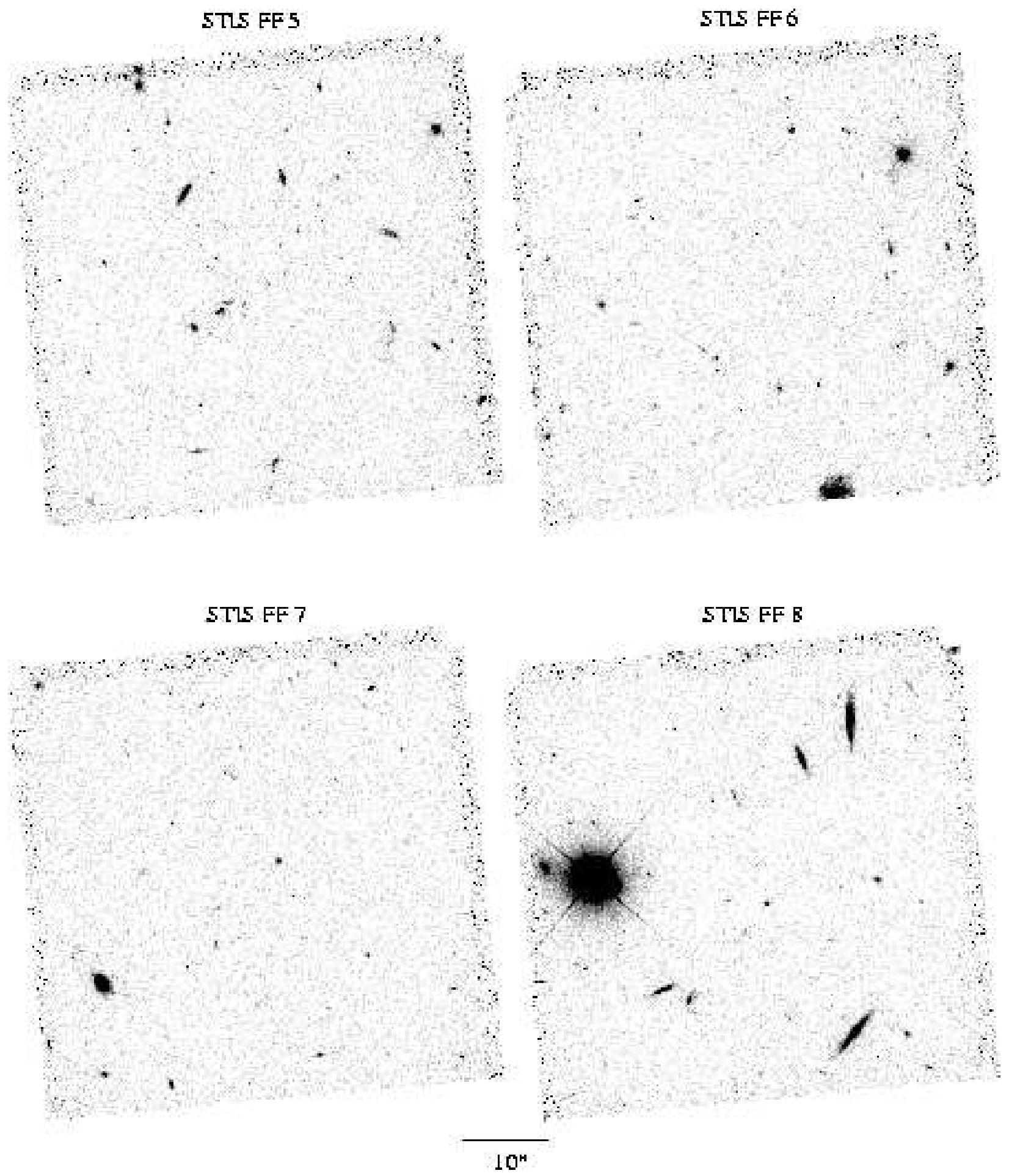

Fig. 7.- STIS flanking fields 5-8. 50CCD, 5100s. North is up, East is to the left. 

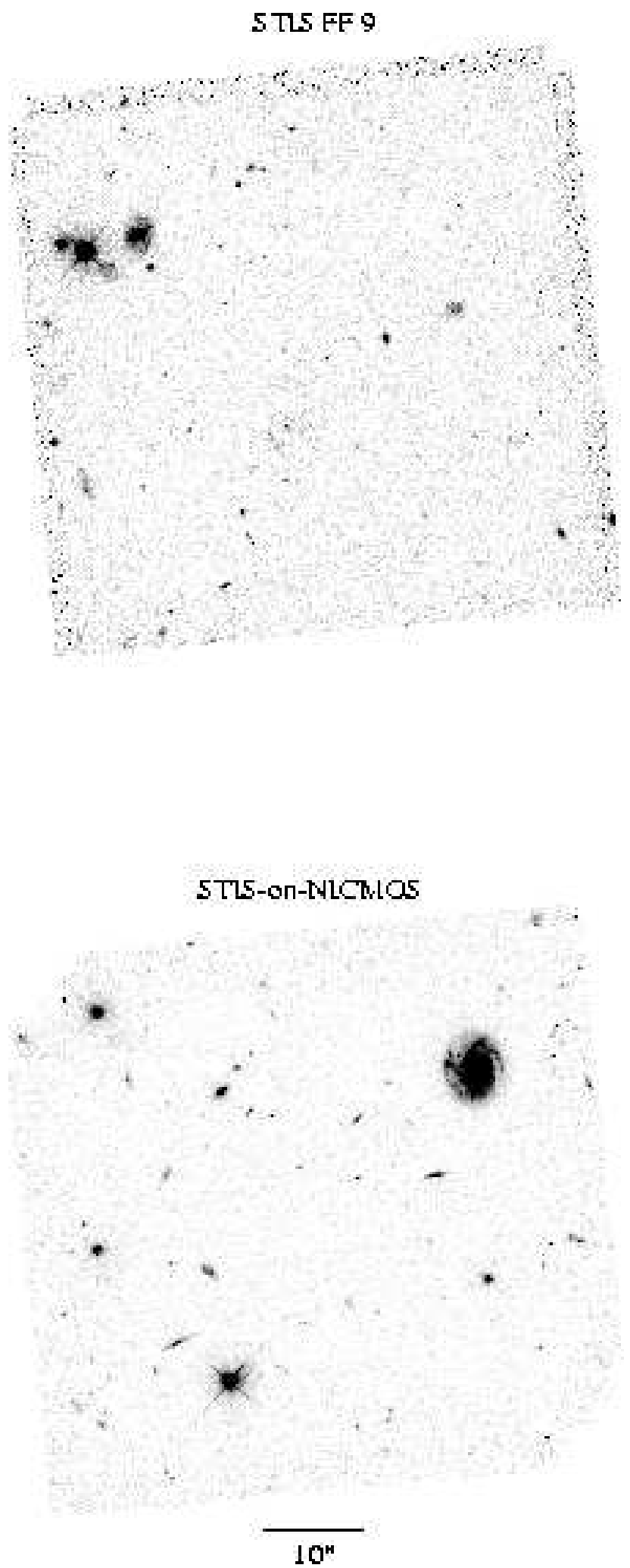

Fig. 8.- Top: STIS flanking field 9. 50CCD, 5100s. Bottom: STIS-on-NICMOS flanking field, 50CCD, 25,900s. North is up, East is to the left. 

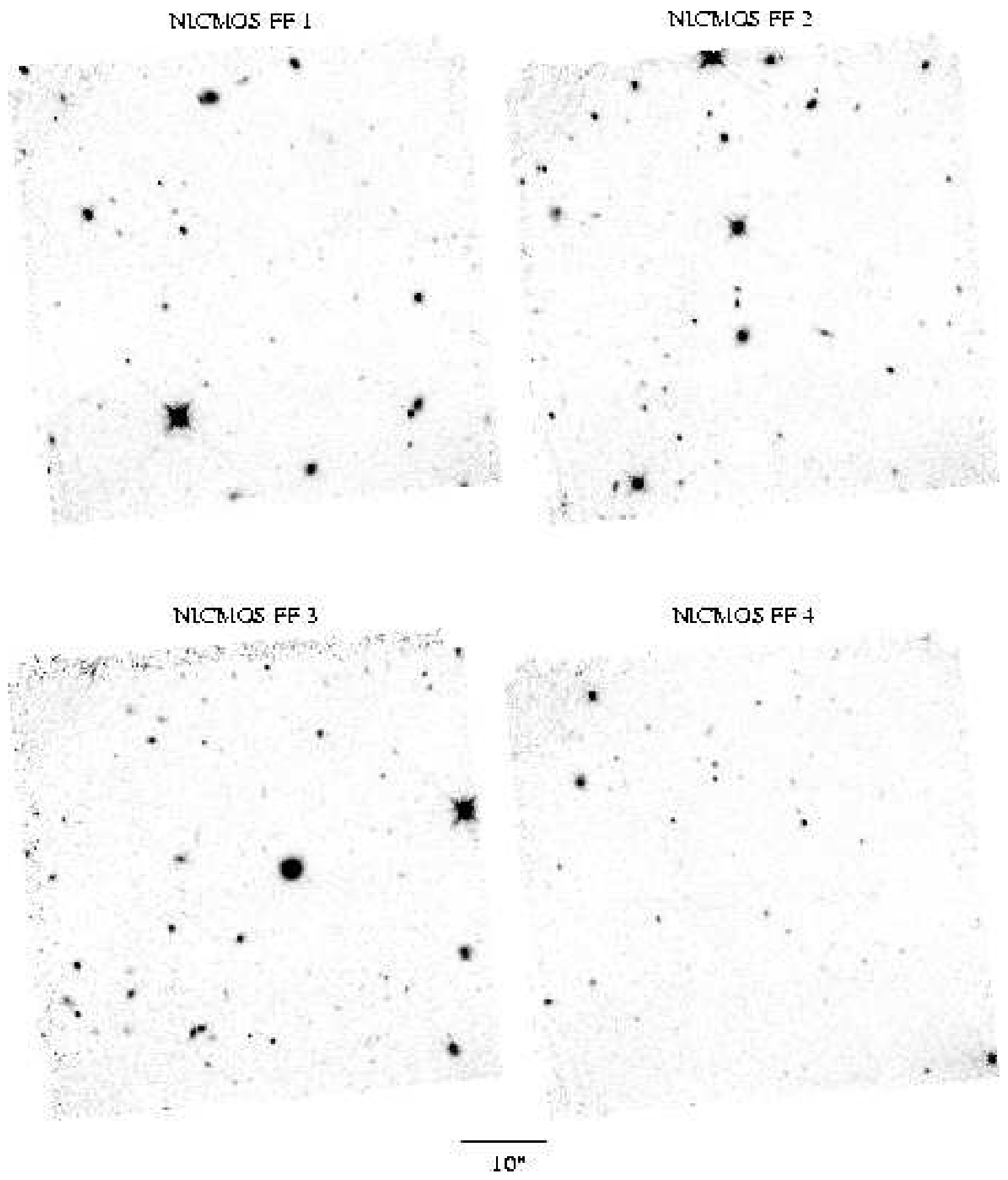

Fig. 9.- NICMOS flanking fields 1-4. NIC3 F160W, 5376s. North is up, East is to the left. 

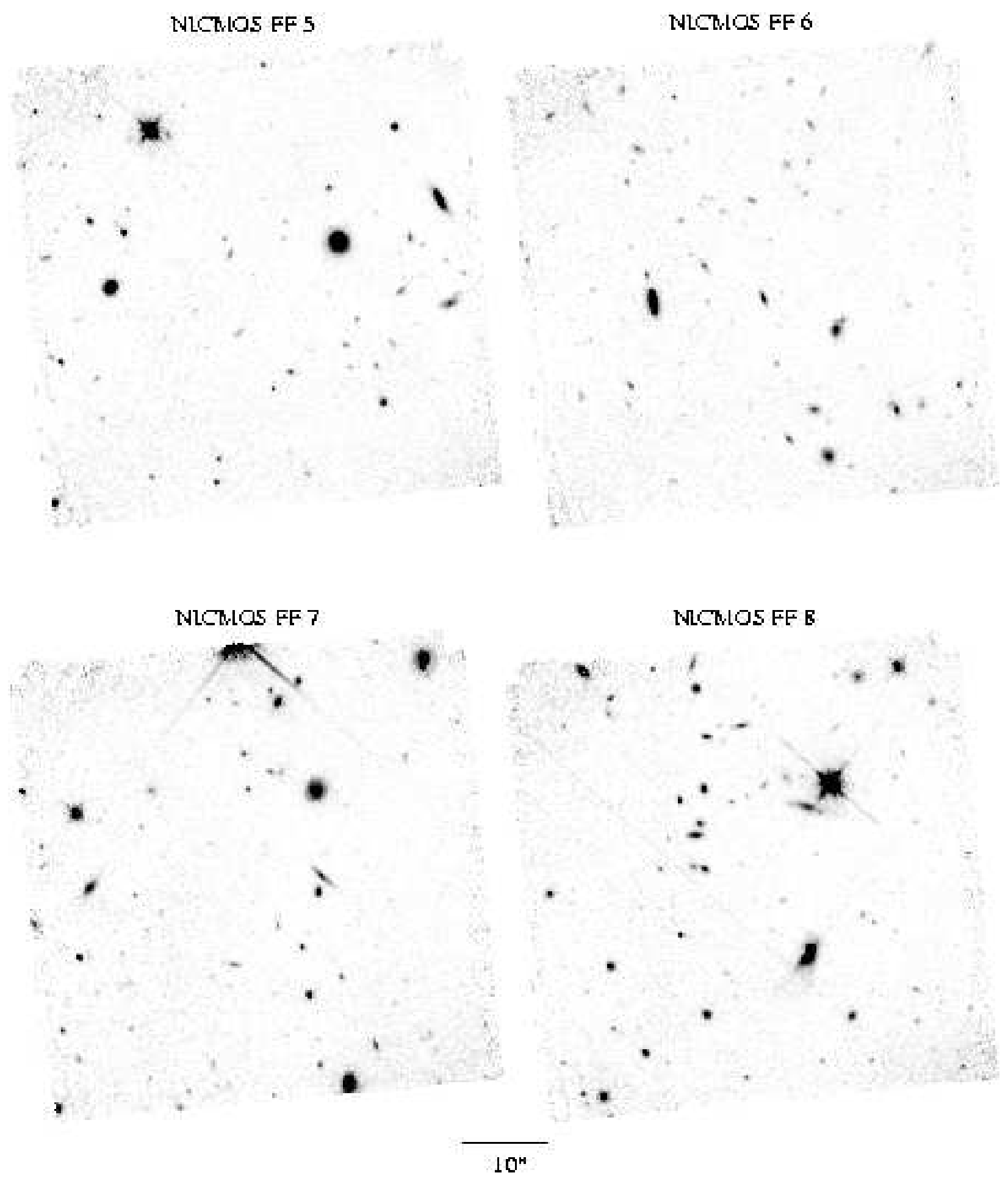

Fig. 10.- NICMOS flanking fields 5-8. NIC3 F160W, 5376s. North is up, East is to the left. 


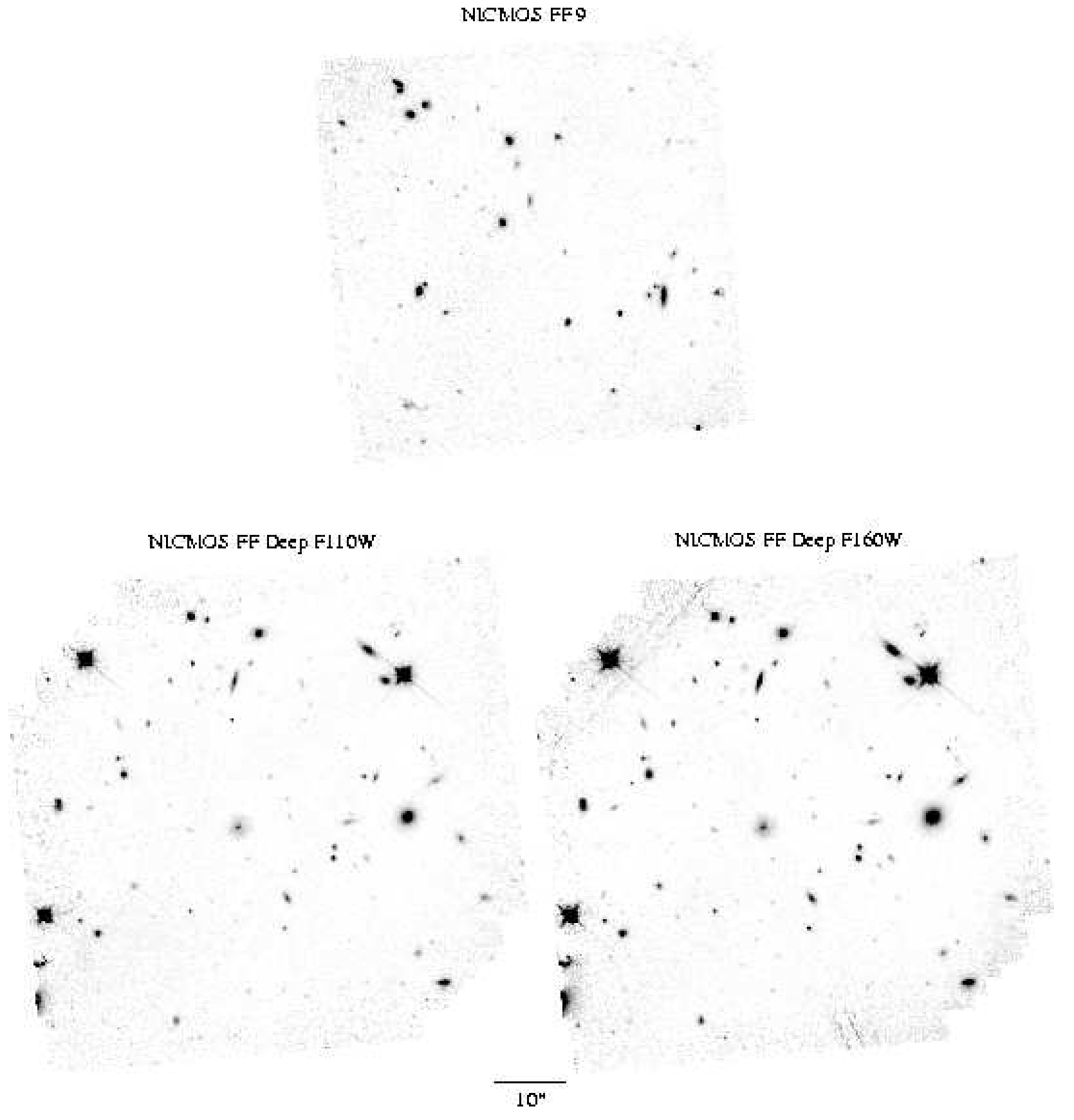

Fig. 11.- Top: NICMOS flanking field 9. NIC3 F160W, 5376s. Bottom: NICMOS deep flanking field. F110W on the left, F160W on the right, both NIC3, 13119s. North is up, East is to the left. Note change in scale between Figure 11 and Figures 9 and 10. 


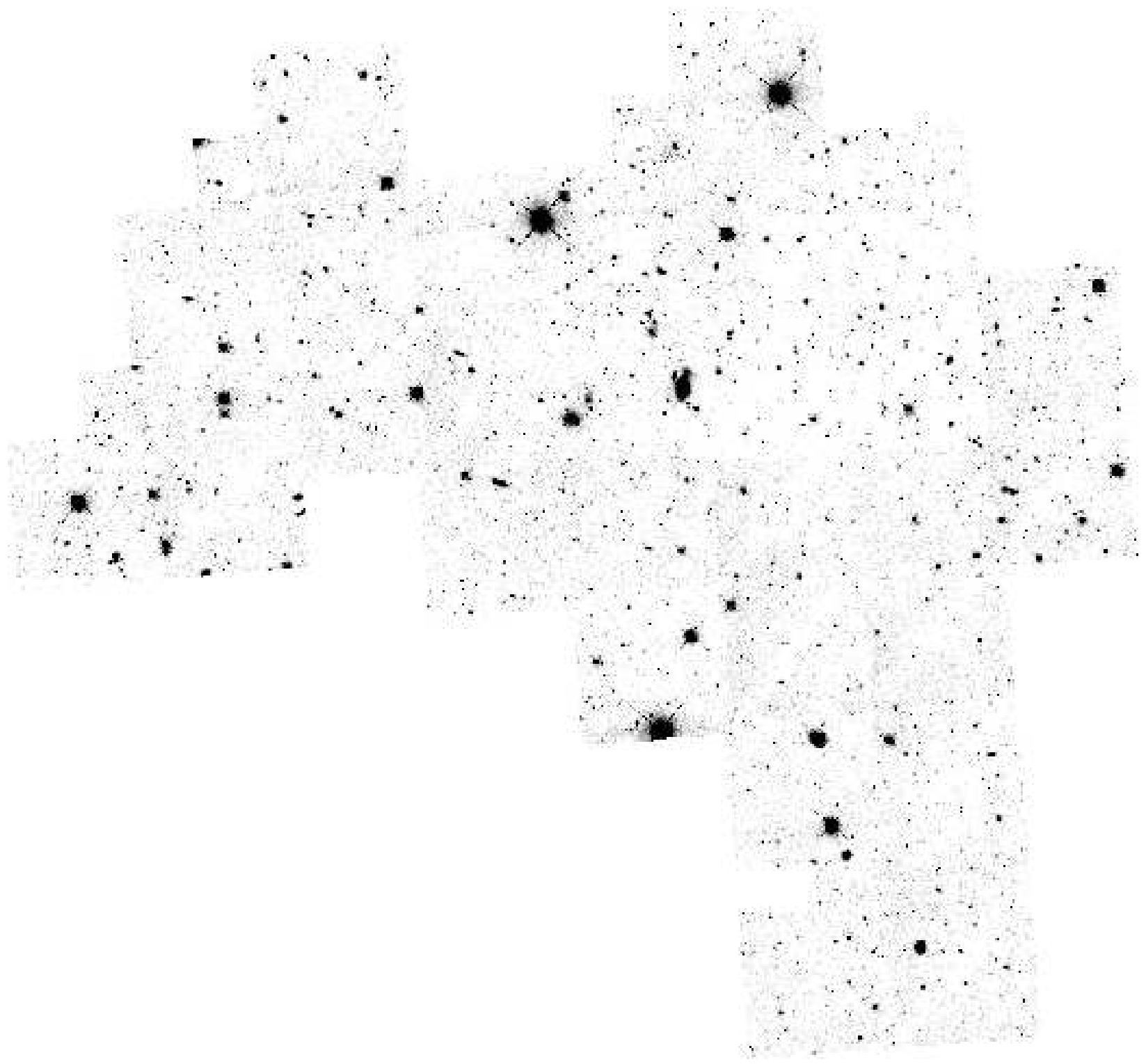

Fig. 12. - The mosaic of contiguous WFPC2 flanking fields 1-9 plus the WFPC2 deep field embedded in it. North is up, East is to the left. 


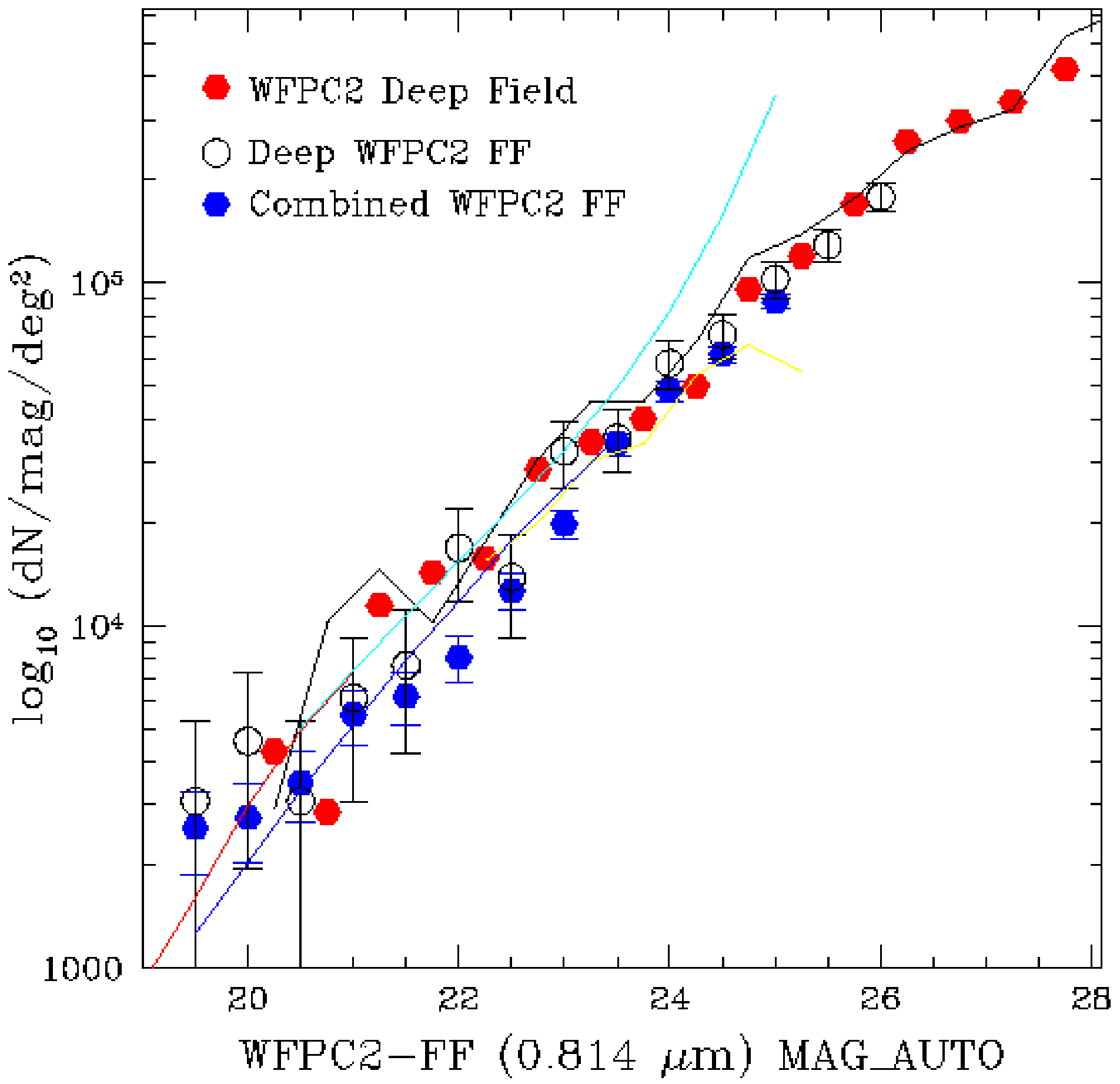

Fig. 13.- Number counts for the HDF-S WFPC2 deep field, the deep F814W (STIS-onNICMOS) WFPC2 parallel flanking field, and for the 9 combined 2-orbit WFPC2 F814W FFs. Also overplotted are the number counts from Hall (1984) in blue, Koo (1986) in red, Tyson (1988) in cyan, Lilly et al. (1991) in yellow, and Gardner et al. (1996) in green. 


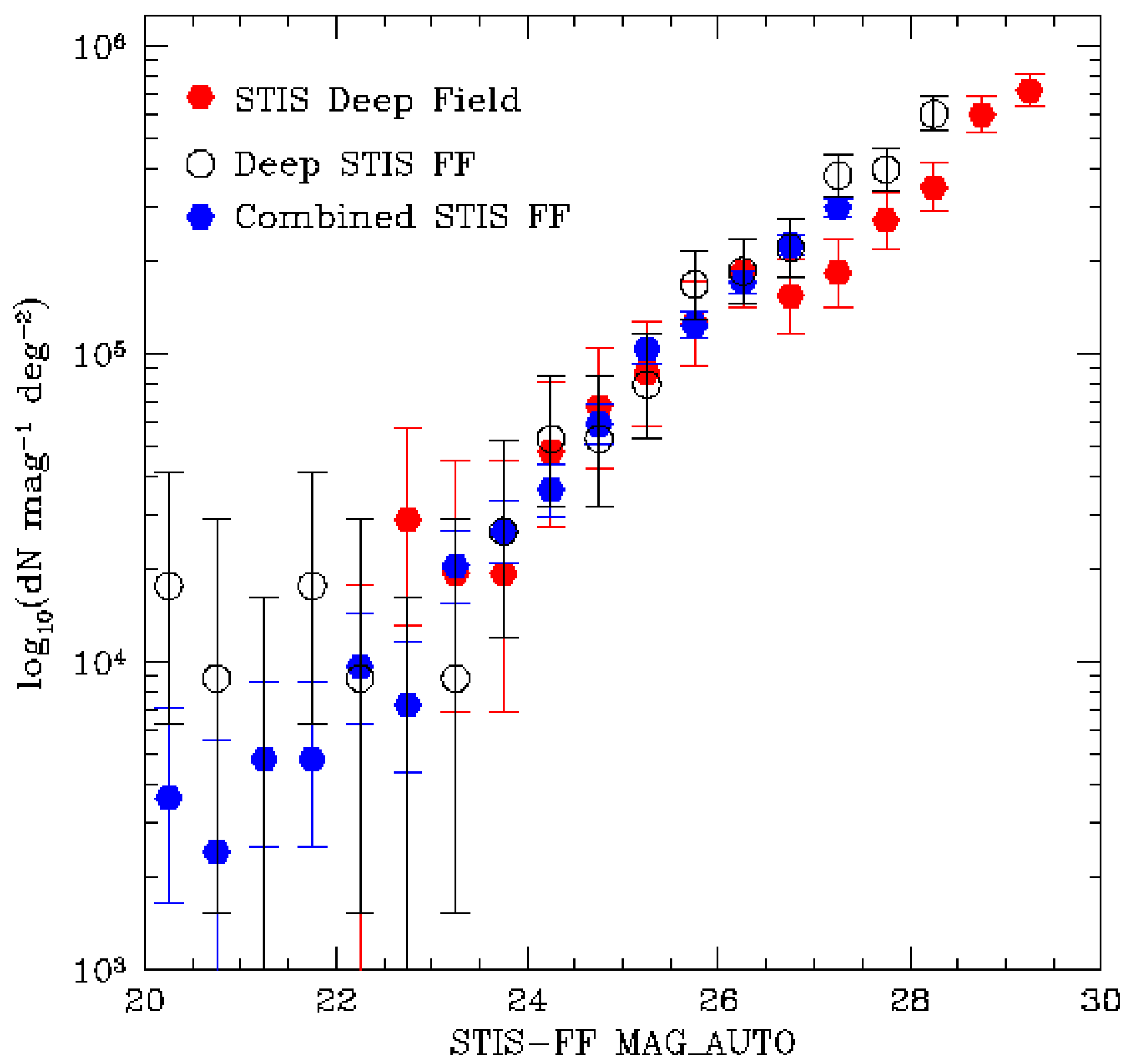

Fig. 14.- Number counts for the STIS 50CCD clear images of the HDF-S STIS deep field, the deep, unconvolved STIS flanking field (STIS- on-NICMOS), and the 9 combined 2-orbit STIS FFs. 


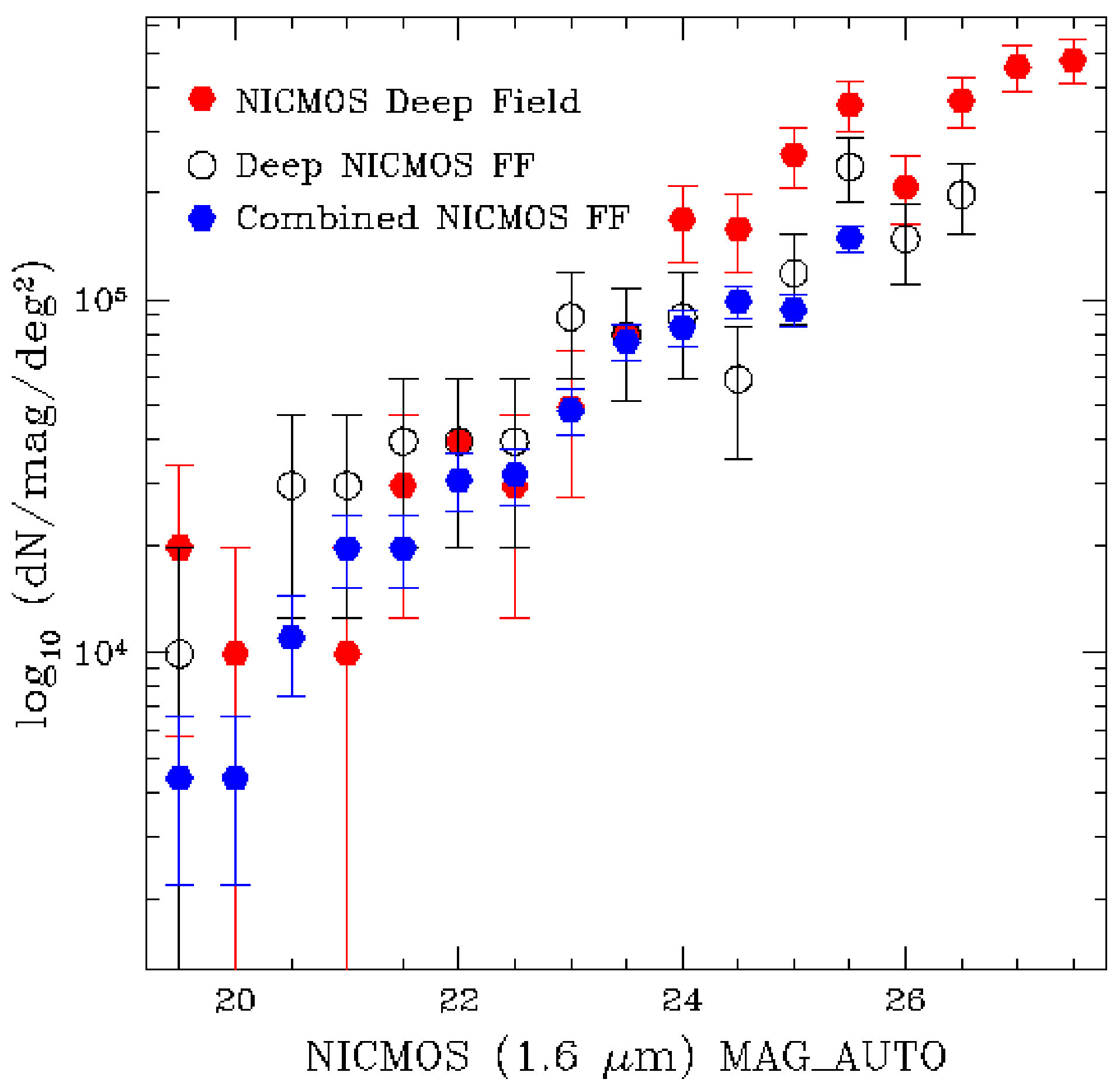

Fig. 15.- Number counts for the HDF-S NICMOS deep field, the deep F160W (STIS-onNICMOS) NICMOS parallel flanking field, and the 9 combined 2-orbit NICMOS F160W FFs. 
Table 1. HDF-South WFPC2 Flanking Fields

\begin{tabular}{lcllrc}
\hline \hline & \multicolumn{2}{c}{ Equatorial } & & & \multicolumn{2}{c}{ Total } \\
Field & RA and Dec $(J 2000)^{\mathrm{a}}$ & UT Date & Filter & $\mathrm{N}_{\exp }$ & $\mathrm{T}_{\exp }(\mathrm{s})$ \\
\hline W1 & 22:33:06.28 -60:31:47.0 & 27 Sep 1998 & F814W & 4 & 5100 \\
W2 & 22:32:45.71 -60:33:59.1 & 09 Oct 1998 & F814W & 4 & 5100 \\
W3 & $22: 33: 14.34-60: 34: 19.5$ & 27 Sep 1998 & F814W & 4 & 5100 \\
W4 & $22: 33: 24.47-60: 33: 10.6$ & 10 Oct 1998 & F814W & 4 & 5100 \\
W5 & $22: 33: 03.81-60: 35: 24.5$ & 11 Oct 1998 & F814W & 4 & 5100 \\
W6 & $22: 32: 53.27-60: 36: 27.9$ & 11 Oct 1998 & F814W & 4 & 5100 \\
W7 & $22: 33: 34.91-60: 32: 07.6$ & 10 Oct 1998 & F814W & 4 & 5100 \\
W8 & $22: 33: 42.61-60: 34: 05.1$ & 11 Oct 1998 & F814W & 4 & 5100 \\
W9 & $22: 32: 52.25-60: 38: 04.7$ & 12 Oct 1998 & F814W & 4 & 5100 \\
WdpV & $22: 32: 09.73-60: 38: 09.6$ & 29 Oct 1998 & F606W & 8 & 9400 \\
WdpI & $22: 32: 09.74-60: 38: 09.8$ & 14 Oct 1998 & F814W & 10 & 11800 \\
\hline
\end{tabular}

${ }^{a}$ Coordinates are for center of final drizzled 4096x4096 image. 
Table 2. HDF-South STIS Flanking Fields

\begin{tabular}{|c|c|c|c|c|c|}
\hline Field & $\begin{array}{c}\text { Equatorial } \\
\text { RA and Dec }(\mathrm{J} 2000)^{\mathrm{a}}\end{array}$ & UT Date & Filter & $\mathrm{N}_{\exp }$ & $\begin{array}{c}\text { Total } \\
\mathrm{T}_{\exp }(\mathrm{s})\end{array}$ \\
\hline S1 & $22: 33: 47.62-60: 32: 20.8$ & 27 Sep 1998 & $50 \mathrm{CCD}$ & 8 & 5100 \\
\hline S2 & $22: 33: 27.10-60: 34: 32.9$ & 09 Oct 1998 & $50 \mathrm{CCD}$ & 8 & 5100 \\
\hline S3 & 22:33:55.73 -60:34:53.4 & 27 Sep 1998 & $50 \mathrm{CCD}$ & 8 & 5100 \\
\hline S4 & 22:34:05.84 -60:33:44.4 & 10 Oct 1998 & $50 \mathrm{CCD}$ & 8 & 5100 \\
\hline S5 & $22: 33: 45.23-60: 35: 58.4$ & 11 Oct 1998 & $50 \mathrm{CCD}$ & 8 & 5100 \\
\hline S6 & 22:33:34.71 -60:37:01.7 & 11 Oct 1998 & $50 \mathrm{CCD}$ & 8 & 5100 \\
\hline S7 & $22: 34: 16.25-60: 32: 41.4$ & 10 Oct 1998 & $50 \mathrm{CCD}$ & 8 & 5100 \\
\hline S8 & 22:34:23.99 -60:34:38.9 & 11 Oct 1998 & $50 \mathrm{CCD}$ & 8 & 5100 \\
\hline S9 & 22:33:33.72 -60:38:38.6 & 12 Oct 1998 & $50 \mathrm{CCD}$ & 8 & 5100 \\
\hline S10 (STIS-on-NIC) & $22: 32: 51.69-60: 38: 48.9$ & 14,29 Oct 1998 & $50 \mathrm{CCD}$ & 18 & 25900 \\
\hline
\end{tabular}

${ }^{a}$ Coordinates are for center of final drizzled 2400x2400 image. 
Table 3. HDF-South NICMOS Flanking Fields

\begin{tabular}{lcrlrr}
\hline \hline & \multicolumn{2}{c}{ Equatorial } & & \multicolumn{2}{c}{ Total } \\
Field & RA and Dec $(J 2000)^{\mathrm{a}}$ & \multicolumn{1}{c}{ UT Date } & Filter & $\mathrm{N}_{\exp }$ & $\mathrm{T}_{\exp }(\mathrm{s})$ \\
\hline N1 & 22:33:02.20 -60:37:39.1 & 27 Sep 1998 & F160W & 4 & 5376 \\
N2 & $22: 32: 41.61-60: 39: 51.1$ & 09 Oct 1998 & F160W & 4 & 5376 \\
N3 & $22: 33: 10.25-60: 40: 11.5$ & 27 Sep 1998 & F160W & 4 & 5376 \\
N4 & $22: 33: 20.38-60: 39: 02.7$ & 10 Oct 1998 & F160W & 4 & 5376 \\
N5 & $22: 32: 59.71-60: 41: 16.6$ & 11 Oct 1998 & F160W & 4 & 5376 \\
N6 & $22: 32: 49.16-60: 42: 19.8$ & 11 Oct 1998 & F160W & 4 & 5376 \\
N7 & $22: 33: 30.81-60: 37: 59.5$ & 10 Oct 1998 & F160W & 4 & 5376 \\
N8 & $22: 33: 38.52-60: 39: 57.1$ & 11 Oct 1998 & F160W & 4 & 5376 \\
N9 & $22: 32: 48.10-60: 43: 56.7$ & 12 Oct 1998 & F160W & 4 & 5376 \\
NdpJ & $22: 32: 05.98-60: 44: 06.1$ & 14,29 Oct 1998 & F110W & 9 & 13119 \\
NdpH & $22: 32: 05.98-60: 44: 06.1$ & 14,29 Oct 1998 & F160W & 9 & 13119 \\
\hline
\end{tabular}

${ }^{a}$ Coordinates are for center of final drizzled 1024x1024 image. 
Table 4. Estimated Limiting Magnitudes

\begin{tabular}{llll}
\hline \hline \multicolumn{1}{c}{ Field } & \multicolumn{1}{c}{ Sample } & Mean & $\sigma$ \\
\hline W1 (WFPC2-FF1) & 18 Objects & 26.05 & 0.45 \\
W2 (WFPC2-FF2) & 9 Objects & 25.87 & 0.48 \\
W3 (WFPC2-FF3) & 7 Objects & 25.92 & 0.70 \\
W4 (WFPC2-FF4) & 15 Objects & 25.92 & 0.62 \\
W5 (WFPC2-FF5) & 11 Objects & 26.02 & 0.35 \\
W6 (WFPC2-FF6) & 14 Objects & 26.04 & 0.42 \\
W7 (WFPC2-FF7) & 10 Objects & 26.04 & 0.33 \\
W8 (WFPC2-FF8) & 19 Objects & 25.98 & 0.33 \\
W9 (WFPC2-FF9) & 10 Objects & 26.32 & 0.31 \\
WFPC2-FF1-9-Avg & 9 Fields & 26.02 & 0.13 \\
WdpV (WFPC2-FF-DEEP-V) & 64 Objects & 27.79 & 0.20 \\
WdpI (WFPC2-FF-DEEP-I) & 152 Objects & 27.15 & 0.26 \\
WFPC2-FF-DEEP-VI-Sum & 12 Objects & 27.13 & 0.19 \\
S1 (STIS-FF1) & 37 Objects & 28.18 & 0.16 \\
S2 (STIS-FF2) & 42 Objects & 28.21 & 0.17 \\
S3 (STIS-FF3) & 44 Objects & 28.23 & 0.15 \\
S4 (STIS-FF4) & 53 Objects & 28.20 & 0.15 \\
S5 (STIS-FF5) & 42 Objects & 28.20 & 0.16 \\
S6 (STIS-FF6) & 38 Objects & 28.32 & 0.16 \\
S7 (STIS-FF7) & 28 Objects & 28.31 & 0.17 \\
S8 (STIS-FF8) & 50 Objects & 28.24 & 0.17 \\
S9 (STIS-FF9) & 41 Objects & 28.23 & 0.17 \\
STIS-FF1-9-Avg & 9 Fields & 28.24 & 0.05 \\
S10 (STIS-on-NIC) & 92 Objects & 29.09 & 0.26 \\
N1 (NICMOS-FF1) & 21 Objects & 26.41 & 0.45 \\
N2 (NICMOS-FF2) & 19 Objects & 26.45 & 0.40 \\
N3 (NICMOS-FF3) & 50 Objects & 25.85 & 0.60 \\
N4 (NICMOS-FF4) & 12 Objects & 26.27 & 0.45 \\
N5 (NICMOS-FF5) & 10 Objects & 26.32 & 0.46 \\
N6 (NICMOS-FF6) & 10 Objects & 26.45 & 0.26 \\
N7 (NICMOS-FF7) & 21 Objects & 26.17 & 0.56 \\
& & &
\end{tabular}


Table 4-Continued

\begin{tabular}{llll}
\hline \hline \multicolumn{1}{c}{ Field } & Sample & Mean & $\sigma$ \\
\hline N8 (NICMOS-FF8) & 11 Objects & 26.57 & 0.29 \\
N9 (NICMOS-FF9) & 18 Objects & 26.44 & 0.25 \\
NICMOS-FF1-9-Avg & 9 Fields & 26.32 & 0.22 \\
NdpJ (NICMOS-DEEP-J) & 29 Objects & 26.94 & 0.51 \\
NdpH (NICMOS-DEEP-H) & 35 Objects & 26.66 & 0.63 \\
NICMOS-DEEP-JH-Sum & 25 Objects & 27.02 & 0.61 \\
\hline
\end{tabular}

Table 5. Non-Default SExtractor Catalog Parameters Used

\begin{tabular}{lllll}
\hline \hline \multicolumn{1}{c}{ Parameter } & WFPC2 & NICMOS & STIS & STIS-on-NICMOS \\
\hline BACK_SIZE & 80 & 40 & 60 & 60 \\
BACK_FILTERSIZE & 3 & 3 & 3 & 3 \\
FILTER_NAME ( " FWHM & 0.18 & 0.21 & 0.085 & 0.085 \\
DETECT_MINAREA & 16 & 16 & 16 & 16 \\
DETECT_THRESH & 1.25 & 0.75 & 0.85 & 0.85 \\
DEBLEND_MIN & 0.03 & 0.002 & 0.1 & 0.035 \\
DEBLEND_NTHRESH & 32 & 32 & 32 & 32 \\
CLEAN & 1 & 1 & 1 & 1 \\
BACKPHOTO_THICK & 25 & 13 & 40 & 40 \\
SEEING_FWHM $(")$ & 0.14 & 0.21 & 0.085 & 0.085 \\
\hline
\end{tabular}


Table 6. Sample of the Object Catalog for the HDF-S WFPC2 Flanking Fields

\begin{tabular}{|c|c|c|c|c|c|c|c|c|c|c|c|c|}
\hline$\overline{\text { Field }}$ & $\overline{\text { Dup }}$ & $\overline{\mathrm{ID}}$ & "HDFS_. & $2 \mathrm{r}-60 \mathrm{~d}$ & $\mathrm{x}$ & $\mathrm{y}$ & $m_{I}$ & $\sigma\left(m_{I}\right)$ & $m_{a I}$ & $r_{h}$ & $\mathrm{~s} / \mathrm{g}$ & Flags $^{a}$ \\
\hline$\overline{\mathrm{W} 2}$ & $\overline{0}$ & 362 & 3242.47 & 3505.7 & 2528.41 & 711.02 & 25.91 & 0.12 & 25.79 & 73 & 0.95 & \\
\hline W6 & 0 & 270 & 3242.47 & 3715.0 & 3644.70 & 1103.99 & 25.54 & 0.09 & 23.27 & 712 & 0.00 & ad \\
\hline W6 & 0 & 236 & 3242.59 & 3706.7 & 3626.60 & 1271.90 & 26.63 & 0.13 & 26.11 & 121 & 0.03 & $\mathrm{~d}$ \\
\hline W9 & 1 & 47 & 3242.85 & 3717.0 & 3436.45 & 3008.71 & 26.40 & 0.11 & 25.51 & 186 & 0.00 & \\
\hline W6 & 2 & 284 & 3242.85 & 3717.1 & 3588.84 & 1062.22 & 26.82 & 0.13 & 25.25 & 307 & 0.00 & \\
\hline
\end{tabular}

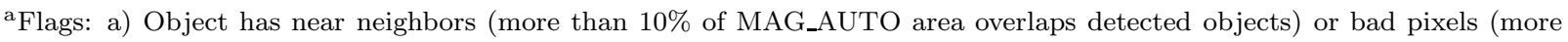
than $10 \%$ of the integrated area); b) Object was originally blended with another; c) At least one pixel is (or very close to) saturated; d) Object is off the image, or within about 30 pixels of the image border.

Note. - The complete version of this table containing both many more objects and many more columns including data from V-band is in the electronic edition of the journal. The printed edition contains only a sample.

Table 7. Sample of the Object Catalog for the HDF-S STIS Flanking Fields.

\begin{tabular}{|c|c|c|c|c|c|c|c|c|c|c|c|c|}
\hline$\overline{\text { Field }}$ & $\overline{\text { Dup }}$ & ID & HDFS_J & $2 \mathrm{r}-60 \mathrm{~d}$ & $\mathrm{x}$ & $\mathrm{y}$ & $m$ & $\sigma(m)$ & $m_{a}$ & $r_{h}$ & $\mathrm{~s} / \mathrm{g}$ & $\bar{F}$ lags $^{\mathrm{a}}$ \\
\hline $\mathrm{S} 10$ & 0 & 271 & 3247.80 & 3855.2 & 2391.80 & 996.95 & & & 29.33 & 52 & 0.920 & $\bar{d}$ \\
\hline S10 & 0 & 288 & 3247.88 & 3858.0 & 2369.78 & 884.81 & 29.81 & 0.17 & 29.05 & 88 & 0.910 & d \\
\hline S10 & 3 & 340 & 3247.89 & 3904.3 & 2367.38 & 634.27 & 28.85 & 0.12 & 28.58 & 75 & 0.110 & $\mathrm{~d}$ \\
\hline S10 & 3 & 234 & 3247.97 & 3851.1 & 2341.77 & 1162.39 & 28.72 & 0.09 & 28.29 & 100 & 0.010 & d \\
\hline S10 & 3 & 323 & 3247.99 & 3902.2 & 2335.56 & 716.30 & 29.05 & 0.12 & 28.19 & 127 & 0.010 & d \\
\hline
\end{tabular}

aFlags: a) Object has near neighbors (more than 10\% of mag_auto area overlaps detected objects) or bad pixels (more than $10 \%$ of th e integrated area); b) Object was originally blended with another; c) At least one pixel is (or very close to) saturated; d) Object is off the image, or within about 30 pixels of the image border.

Note. - The complete version of this table containing both many more objects and many more columns is in the electronic edition of the journal. The printed edition contains only a sample.

Table 8. Sample of the Object Catalog for the HDF-S NICMOS Flanking Fields.

\begin{tabular}{|c|c|c|c|c|c|c|c|c|c|c|c|c|}
\hline Field & Dup & $\overline{\mathrm{ID}}$ & HDFS_J & $2 \mathrm{r}-60 \mathrm{~d}$ & $\mathrm{x}$ & $\mathrm{y}$ & $m_{H}$ & $\sigma\left(m_{H}\right)$ & $m_{a H}$ & $r_{h}$ & $\mathrm{~s} / \mathrm{g}$ & 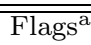 \\
\hline NdpHJ & 0 & 53 & 3201.26 & 4413.6 & $\begin{array}{c}62.63 \\
\end{array}$ & 655.99 & 25.23 & 0.15 & 24.21 & & 0.990 & $\mathrm{ad}$ \\
\hline NdpHJ & 0 & 30 & 3201.92 & 4422.7 & 138.27 & 770.43 & 26.34 & 0.21 & 25.59 & 208 & 0.290 & $\mathrm{~d}$ \\
\hline NdpHJ & 0 & 38 & 3201.95 & 4418.5 & 135.69 & 714.68 & 22.48 & 0.02 & 22.37 & 500 & 0.030 & $\mathrm{~d}$ \\
\hline NdpHJ & 0 & 78 & 3202.00 & 4405.8 & 124.10 & 545.56 & 27.45 & 0.41 & 26.12 & 206 & 0.050 & $\mathrm{~d}$ \\
\hline NdpHJ & 0 & 18 & 3202.25 & 4427.2 & 176.32 & 826.65 & 26.03 & 0.22 & 24.89 & 313 & 0.100 & $\mathrm{~d}$ \\
\hline
\end{tabular}

${ }^{a}$ Flags: a) Object has near neighbors (more than $10 \%$ of mag_auto area overlaps detected objects) or bad pixels (more than $10 \%$ of th e integrated area); b) Object was originally blended with another; c) At least one pixel is (or very close to) saturated; d) Object is off the image, or within about 30 pixels of the image border.

Note. - The complete version of this table containing both many more objects and many more columns including data from J-band is in the electronic edition of the journal. The printed edition contains only a sample. 